Article

\title{
Rapid Mapping and Annual Dynamic Evaluation of Quality of Urban Green Spaces on Google Earth Engine
}

\author{
Qiang Chen (D, Cuiping Zhong, Changfeng Jing*(D), Yuanyuan Li, Beilei Cao and Qianhao Cheng
}

check for

updates

Citation: Chen, Q.; Zhong, C.; Jing, C.; Li, Y.; Cao, B.; Cheng, Q. Rapid Mapping and Annual Dynamic Evaluation of Quality of Urban Green Spaces on Google Earth Engine. ISPRS Int. J. Geo-Inf. 2021, 10, 670. https://doi.org/10.3390/ijgi10100670

Academic Editors: Christos Chalkias, Vassilis Pappas, Andreas Tsatsaris and Wolfgang Kainz

Received: 29 July 2021

Accepted: 28 September 2021

Published: 2 October 2021

Publisher's Note: MDPI stays neutral with regard to jurisdictional claims in published maps and institutional affiliations.

Copyright: (c) 2021 by the authors. Licensee MDPI, Basel, Switzerland. This article is an open access article distributed under the terms and conditions of the Creative Commons Attribution (CC BY) license (https:// creativecommons.org/licenses/by/ $4.0 /)$.
School of Geomatics and Urban Spatial Information, Beijing University of Civil Engineering and Architecture, Beijing 102616, China; chenqiang@bucea.edu.cn (Q.C.); 2108521519021@stu.bucea.edu.cn (C.Z.); 2108570020085@stu.bucea.edu.cn (Y.L.); 2108570020113@stu.bucea.edu.cn (B.C.); 2108521519015@stu.bucea.edu.cn (Q.C.)

* Correspondence: jingcf@bucea.edu.cn; Tel.: +86-10-6120-9335

\begin{abstract}
In order to achieve the United Nations 2030 Sustainable Development Goals (SDGs) related to green spaces, monitoring dynamic urban green spaces (UGSs) in cities around the world is crucial. Continuous dynamic UGS mapping is challenged by large computation, time consumption, and energy consumption requirements. Therefore, a fast and automated workflow is needed to produce a high-precision UGS map. In this study, we proposed an automatic workflow to produce up-to-date UGS maps using Otsu's algorithm, a Random Forest (RF) classifier, and the migrating training samples method in the Google Earth Engine (GEE) platform. We took the central urban area of Beijing, China, as the study area to validate this method, and we rapidly obtained an annual UGS map of the central urban area of Beijing from 2016 to 2020. The accuracy assessment results showed that the average overall accuracy (OA) and kappa coefficient (KC) were $96.47 \%$ and $94.25 \%$, respectively. Additionally, we used six indicators to measure quality and temporal changes in the UGS spatial distribution between 2016 and 2020. In particular, we evaluated the quality of UGS using the urban greenness index (UGI) and Shannon's diversity index (SHDI) at the pixel level. The experimental results indicate the following: (1) The UGSs in the center of Beijing increased by $48.62 \mathrm{~km}^{2}$ from 2016 to 2020, and the increase was mainly focused in Chaoyang, Fengtai, and Shijingshan Districts. (2) The average proportion of relatively high and above levels (UGI > 0.5) in six districts increased by $2.71 \%$ in the study area from 2016 to 2020, and this proportion peaked at $36.04 \%$ in 2018 . However, our result revealed that the increase was non-linear during this assessment period. (3) Although there was no significant increase or decrease in SHDI values in the study area, the distribution of the SHDI displayed a noticeable fluctuation in the northwest, southwest, and northeast regions of the study area between 2016 and 2020. Furthermore, we discussed and analyzed the influence of population on the spatial distribution of UGSs. We found that three of the five cold spots were located in the east and southeast of Haidian District. Therefore, the proposed workflow could provide rapid mapping and dynamic evaluation of the quality of UGS.
\end{abstract}

Keywords: urban green spaces; Google Earth Engine (GEE); Random Forest (RF); Sentinel-2; Beijing; dynamic evaluation

\section{Introduction}

Urban green spaces (UGSs) provide a sustainable and high-quality living environment for city dwellers. In recent years, UGSs have played an important role in urban planning and policymaking [1]. UGS refers to all urban open spaces covered with vegetation by design or default [2]. UGSs, including parks, gardens, street trees, urban forests, and historical places, improve the quality of city life in several aspects [3]. UGSs also create a healthy and comfortable natural environment; for example, UGSs can provide ecosystem services (ES) [4] and rich biodiversity [5] and mitigate the urban heat island (UHI) [6]. Furthermore, a considerable body of literature has proven that UGS can influence both 
the physical and mental health of human beings [7-9]. Environmental justice occurs when green space is equally distributed in a city, and this has been discussed worldwide [10]. For city planners, up-to-date and dynamic vegetation distribution assessments will improve the efficacy of their greening efforts [11].

However, previous studies analyzed the change in UGS distribution using two or more Landsat images spaced five or ten years apart. Fan et al. [12] evaluated the spatialtemporal patterns of public green space accessibility in 2000 and 2010 using multi-source satellite imagery, including historical land-use maps, Landsat 5 Thematic Mapper (TM), Landsat 7 Enhanced Thematic Mapper plus (ETM+), and SPOT5. However, they did not provide important insights into the current patterns of urban green spaces in Shanghai, China. Thus, they were unable to provide timely and effective information about UGSs to decision makers. Sathya Kumar et al. [3] demonstrated a spatiotemporal analysis of urban green space distribution at the neighborhood level; the study computed seven UGS distribution indicators for 2001 and 2010 and revealed that UGS in Mumbai had generally diminished, fragmented between 2001 and 2010, but it ignored the dynamic changes in the distribution of UGS.

Annual analyses of green space cover dynamics in urban areas provide a thorough understanding of dynamic changes in the natural environment around urban residences [13]. Monitoring the distribution of UGS at a relatively high spatial-temporal resolution contributes to a further understanding of the quality and accessibility of UGS for urban residents.

However, the relevant literature analyzes the annual distribution and amount of greenspace using the Normalized Difference Vegetation Index (NDVI) time series extracted from the Moderate Resolution Imaging Spectroradiometer (MODIS) [14-16] over land cover maps, particularly if the study area is large or the study is investigating the distribution of UGS over time. NDVI is uniformly calculated from remotely sensed data, such that measures are consistent across time and space. Land cover datasets, on the other hand, capture greenspace at a single time point and are generally updated every few years rather than seasonally or annually.

Although some studies have shown that long-term and multi-source satellite images are helpful for detecting changes in vegetation, utilizing this information to map large areas periodically poses challenges, such as high computation complexity and processing costs [17]. These problems can now be solved by the use of cloud-based platforms for remote sensing data, such as the Google Earth Engine (GEE) platform.

GEE is a free cloud platform that hosts massive free satellite images, such as Landsat TM 7/8; MODIS; and Sentinel-1, -2, -3, and -5-P. GEE and its web-based integrated development environment (IDE) enable rapid and easy prototyping, analysis, and visualization of large-scale geospatial data through parallel processing, which reduces computational time. Many scholars have used GEE in various applications regarding the environment and natural resources, such as wetland inventory [18], land cover mapping [19,20], automatic water detection [21], forest degradation [22], cropland classification [23], vegetation conservation and sustainability [24], flood mapping [25], and other types of mapping and detection applications. For example, Mahdianpari et al. [26] improved the method and results of the first-generation Canadian wetland inventory map at a $10 \mathrm{~m}$ resolution using the GEE platform. Ghorbanian et al. produced an improved Iranian land cover map with a spatial resolution of $10 \mathrm{~m}$ using a multi-temporal synergy of Sentinel-1 and Sentinel-2 satellite datasets [27].

GEE has been used to map and analyze UGS on a global and national scale [28-30]. Furthermore, some studies evaluated changes in forest ecosystems, which can provide valuable information about climate change adaptation, using the GEE platform. A method proposed by Bullock et al. [22] extends previous research in spectral mixture analysis for the identification of forest degradation to the temporal domain, and this approach was applied using the GEE platform. Gilani et al. [30] identified changes in mangrove cover in Pakistan using the Random Forest (RF) classifier available in the GEE geospatial cloud computing platform. However, few studies have evaluated the quality of UGS dynamically using 
GEE. Open satellite datasets with high spatial and temporal resolution, cloud computing platforms, and machine learning algorithms offer new possibilities for mapping changes in urban vegetation. Some studies measured access to UGS by means of data-driven geographical information system (GIS) modeling, which included a series of sub-indicators relevant to the proximity and quality of UGS and its ecosystem services derived from spatial and questionnaire data. Stessens et al. [31] proposed a GIS-based tool to evaluate accessibility to-and the quality of-UGS to support decision making at the urban scale in Brussels. The World Health Organization presented a suggested indicator of accessibility to green space and applied it in three European cities using a geographical information system (GIS)-based approach. However, the lack of sufficient and reliable geographic data has limited the application of GIS in periodic assessments of UGS. A few studies presented remote sensing-based approaches to assess the distribution of UGS at different levels, such as region, city, and neighborhood; several methodological and practical issues hamper the performance of regular and up-to-date assessments. For example, several studies only use quantitative measures, such as the total green space area in a district or the proportion of green area to total urban area. Recent studies highlight the importance of perceived quality in addition to the amount of green space when examining the beneficial effects of green space. Some studies [32] have also revealed that both higher vegetation quality (i.e., the amount of vegetation) and diversity (i.e., different types of vegetation) visible from home are related to health.

The simplest and most commonly used objective measure of natural space is the Normalized Difference Vegetation Index (NDVI), which represents "greenness" rather than "greenspace" due to its method of calculation. Atasoy [33] used NDVI to estimate the most current urban green space density and distribution in Osmaniye city and represented different types of vegetation using an NDVI threshold instead of a classification method. It was proven that using NDVI alone to identify the quality and accessibility of a specific area will produce conflicting results. Recent studies showed that some configurational aspects also influence the quality as perceived by residents, such as size, shape, and fragmentation. Large and well-connected green spaces support greater biodiversity and aesthetic quality. Therefore, configurational aspects extracted from remote sensing data can serve as indicators of green space quality beyond the NDVI. Prior studies measured the quality of UGS merely using indicators of the same category: vegetation indices or configurational indicators. For example, Xie et al. [9] used two vegetation indices, the Normalized Difference Vegetation Index (NDVI) and Enhanced Vegetation Index (EVI), to assess the level of residential greenness and defined long-term greenness exposure as the average of NDVI and EVI during a particular period. Sathya Kumar [3] adopted seven UGS configuration indicators to assess its distribution change between 2001 and 2011. These studies have not comprehensively quantified residents' perceived green space quality, as the remote sensing information has not been fully extracted.

Given the above, we attempt to propose a remote sensing-based approach to analyze the spatiotemporal distribution of UGS annually in GEE. Our research objectives are the following:

- $\quad$ Propose a novel and rapid workflow based on Sentinel-2 images to produce UGS maps with a $10 \mathrm{~m}$ spatial resolution and high accuracy and use the sample migrating method to produce up-to-date UGS maps annually.

- Evaluate the spatiotemporal distribution and quality of UGS at the pixel level from 2016 to 2020 by using six landscape pattern indicators.

\section{Materials and Methods}

\subsection{Study Area and Data}

Beijing is located in the north of China with a central location at $39^{\circ} 56^{\prime} \mathrm{N}$ and $116^{\circ} 20^{\prime} \mathrm{E}$, occupying 16,410 $\mathrm{km}^{2}$. By the end of 2019 and the beginning of 2020, the city's permanent resident population was 21.57 million. The study area covers $1385.35 \mathrm{~km}^{2}$ and is located in the center of Beijing, including six districts: Chaoyang, Dongcheng, Fengtai, Haidian, 
Shijingshan, and Xicheng (Figure 1). The western part comprises mountainous regions, and the east is a plain. These districts have the most prosperous politics, economy, and culture and the densest population. By the end of 2019, approximately $48 \%$ of the total population lived in this highly urbanized region. According to the latest Beijing Master Plan (2016-2035) and Afforestation project (2018-2022), the government has increased the size of green recreation spaces, such as parks, small and microgreen spaces, and activity squares, in order to provide urban residents with more convenient recreational and relaxation spaces.

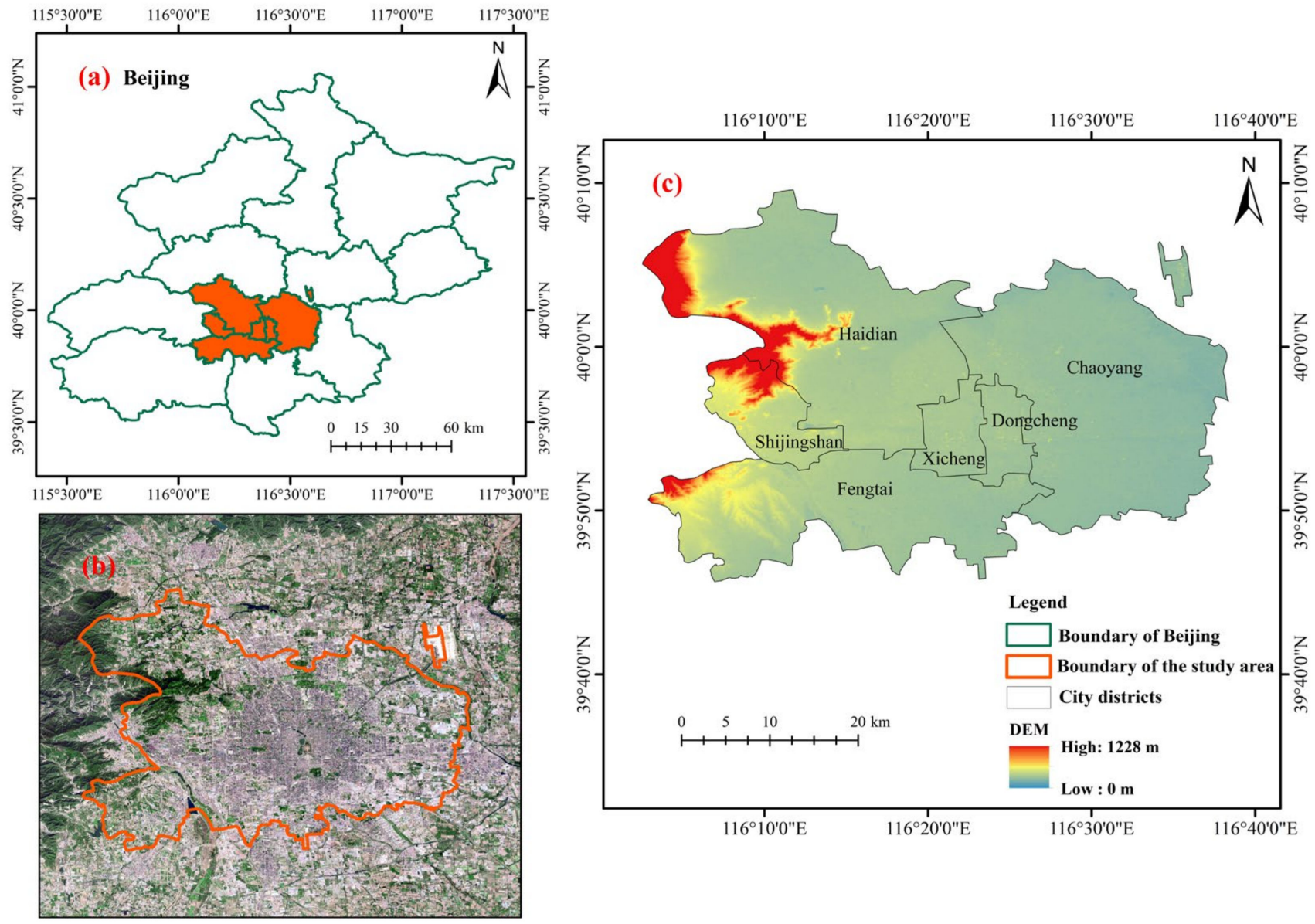

Figure 1. Study area. (a) the study area is located in the center of Beijing; (b) the background image is a Sentinel-2 image captured on 10 August of 2020, projected in WGS84, in true color ( $\mathrm{R}=$ band 4, G = band 3, and B = B2), with a spatial resolution of $10 \mathrm{~m}$; (c) the digital elevation model (DEM) at $30 \mathrm{~m}$ spatial resolution and the distribution of the six districts - the small enclave located in the northeast of the research area is Beijing Capital International Airport, managed by Chaoyang District.

Google Earth Engine's public data archive includes a large amount of historical imagery and scientific datasets, and most of these datasets are image collections, such as Landsat, MODIS, and Sentinel [34]. The concept of ImageCollection proposed by GEE means that an ImageCollection is a stack or sequence of images and can be loaded by pasting an Earth Engine asset ID into the ImageCollection constructor. This concept makes it more convenient for users to search for the images that they need. The high-resolution multispectral images from Sentinel-2 can be used to monitor vegetation, soil and water cover, and land cover change, as well as humanitarian and disaster risk. The level-2A product provided by GEE was computed by running Sen2Cor, which means that this imagery product has been preprocessed for radiation and atmospheric correction. In the GEE code editor environment, the Sentinel-2 level 2A data were imported as an image collection, and then a filtering process was executed to obtain the appropriate seasonal data. In our study, this image collection was filtered by three variables: covered location, 
date, and cloud percentage. The covered location was the polygon of our study area, which we imported into the GEE environment. The date range was from June 21 to September 21, representing the period of the highest growth of plants in summer. Finally, we filtered out the images with cloud cover greater than $7 \%$ by querying the cloudy pixel percentage value stored as metadata for each image. After the data filtering process was completed, we obtained a new image collection of low cloud cover in summer in the study area. To detect and mask out the remaining clouds, the QA60' bitmask band (a quality flag band) available in the metadata of Sentinel-2 imagery was employed. In this study, three bands (B5, B6, and B7, $20 \mathrm{~m}$ ) of Sentinel-2 were resampled to a resolution of $10 \mathrm{~m}$. We used seven multispectral bands (B2-B8) to produce a high-resolution (10 m) UGS map.

\subsection{Methodology}

This section introduces the automatic mapping and dynamic evaluation of the quality of UGS using GEE. The procedures of the automatic mapping of UGS include two parts: multi-feature extraction and machine learning classification (Figure 2) and training sample migration (Figure 3).

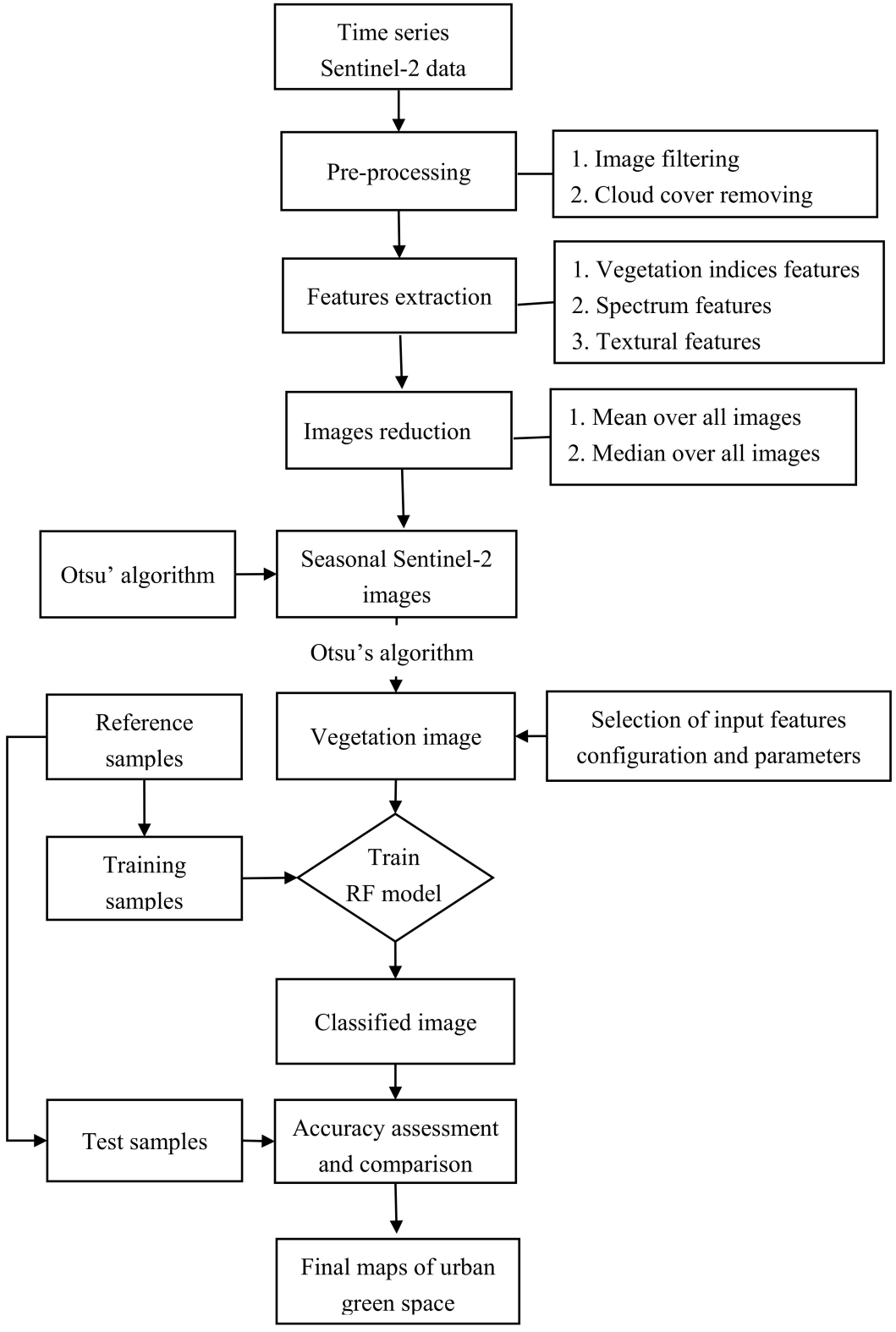

Figure 2. Flowchart of the proposed methodology for UGS classification in GEE. 


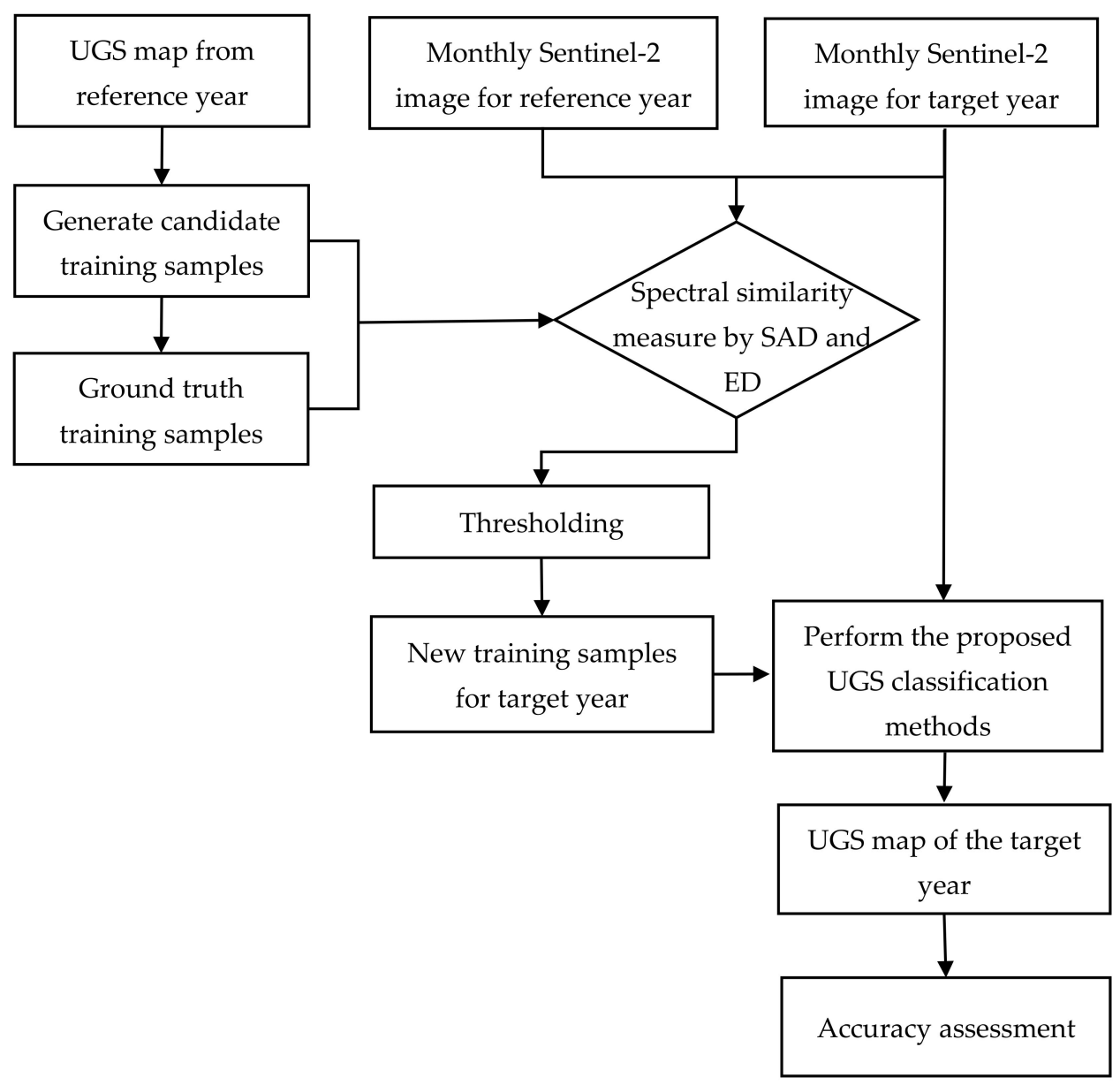

Figure 3. Flowchart to produce up-to-date UGS maps for any target year.

\subsubsection{Extraction and Classification of UGS}

For trees and low plants, such as grasslands, there are different spectral and textural features in multispectral images $[35,36]$. Therefore, we extracted three types of features from the Sentinel-2 images to distinguish between different vegetation types. Considering that forests in mountainous areas cover higher altitudes than the plain areas where urban trees are usually located, we added the elevation band and slope band provided by the $30 \mathrm{~m}$ spatial digital elevation model (DEM) available in GEE.

i. Spectral features and vegetation indices

Typical spectral reflection characteristics of vegetation can be described as the following: in the visible waves, there are two absorption valleys and one reflection peak; in the near-infrared band, there is strong reflectivity in the $0.74-1.3 \mu \mathrm{m}$ range spectrum. The spectral reflectance of different plant species is different in the near-infrared band; the sensitive spectral reflectance changes in vegetation in different growth states in the near-infrared and visible bands are crucial for identifying vegetation types and monitoring the growth of vegetation (Appendix A, Table A1).

Another type of feature that enables the identification and monitoring of vegetation is vegetation indices, which are calculated by multispectral remote sensing data. In general, various vegetation indices can signify the reflection characteristics of vegetation in visible and near-infrared wavelengths. However, different vegetation indices reflect different vegetation growth conditions. We selected six vegetation indices for feature extraction of UGS (Appendix A, Table A2). 


\section{ii. Textural features}

The relevant literature shows that texture is one of the most important characteristics in the classification of objects of interest in a satellite image or an aerial photograph $[37,38]$. The concept of texture refers to the regularity and features formed by the repeated appearance of a large number of small objects in an image. A set of features has been developed for classifying images, and the gray-level co-occurrence matrix is a widely used method for texture feature extraction. The texture metrics can be computed by GEE from the gray-level co-occurrence matrix (GLCM) around each pixel of every band, and this implementation computes the 14 GLCM metrics proposed by Haralick and 4 additional metrics from Conners (Appendix A, Table A3). In our study, we selected the NIR band as the input band to compute the textural features.

\section{iii. Image reduction}

All computed features can be added as a separate band for each image in the summer image collection. An image collection had to be reduced to a single image, containing each selected image's information, to perform the extraction and classification. We obtained a single image by performing a process of reduction. The pixel value of the new image was calculated by a statistical calculation of the pixel value of the same position in the image collection. We used the median, mean, and values of pixels to perform the reduction.

\section{iv. Otsu's algorithm}

In order to differentiate vegetation and non-vegetation categories automatically, Otsu's optimal method [39] was implemented and applied to the NDVI band of the seasonal Sentinel-2 image in our study on the GEE platform. Otsu's method is an automatic, unsupervised image segmentation method based on threshold values. The threshold values were calculated from the grayscale histogram of single-band images by maximizing the between-class variance of the target and background dynamically. Furthermore, Otsu's method is widely used in digital image processing due to its flexibility for different data with different spatial resolutions, and it has been used to extract tidal flats [40] and land surface water [41].

\section{v. Machine learning classification}

Machine learning classification is one of the four main analytic categories of machine learning algorithms adopted for remote sensing data processing [42]. Non-parametric algorithms, such as CART, non-linear SVM, and RF, have been widely used to process a large amount of data with no prior knowledge in a flexible way.

The CART algorithm is a very popular classification method used in remote sensing classification for its efficiency and simplicity in decision making [43]. The distinguishing characteristic of CART is that it makes the final decision by maximizing the value of information gain.

The support vector machine (SVM) is developed based on statistical learning theory, and it improves the generalization capability of the model by reducing the model generalization error while minimizing the sampling error [44].

The characteristics of SVM can be summarized as the following three points: firstly, this approach realizes a non-linear map from the input data into high dimensional feature space, and an optimal classification hyperplane is constructed at the same time. Secondly, SVM is especially suitable for limited sample problems, and the advantage of using SVM is that it has largely solved the problems in traditional algorithms of model selection, overlearning, non-linearity, local minimum point, etc. Finally, this approach improves the efficiency of decision making by automatically choosing the important data points and reducing the number of decision points. The SVM algorithm is a popular classification method used in remote sensing classification for its efficiency and simplicity in decision making [45].

A Random Forest (RF) classifier is a non-parametric classifier that has been used in most classification problems $[46,47]$. The RF classifier is an ensemble classifier that 
combines a set of CARTs to make better classification decisions [48]. Each decision tree is created by randomly selecting a subset of training samples from the original dataset; on this basis, the feature subspace is structured, and the final class decision is made by these decision trees.

The RF classifiers are the most commonly selected in the GEE platform and have obtained a wide range of overall accuracy [34]. There are several important characteristics of RF in the GEE platform. First, the RF algorithm in GEE allows different parameters to be set in order to obtain higher accuracy: the number of decision trees and the number of variables per split, the minimum leaf population, the bag fraction, the maximum number of nodes in each tree, and the randomization seed. Second, a significant advantage of the RF classifier is its capability of determining the importance of input features [49]. The RF variable ranking has been recently added to GEE as an output of the Random Forest classifier [26]. The variable importance (VI) measurement provided by the RF classifier allows for selecting the best combination of features to achieve higher accuracy. In addition, RF does not need to know or assume the distribution of the data in advance and is less sensitive to the quality of training data.

\subsubsection{Accuracy Assessment of UGS Maps}

In order to better represent the acquired classification maps, a statistical measure of accuracy for validation is essential. In this study, we used the confusion matrix of the classification results to assess the statistical accuracy by four indicators computed from the confusion matrix, including the overall accuracy (OA), the producer's accuracy (PA), the user's accuracy (UA), and the kappa coefficient (KC). The confusion matrix is a table that contains the number of pixels in each class that were classified correctly or incorrectly, and the computation of the indicators mentioned above is allowed [50].

The OA is calculated by the ratio of the number of correctly classified pixels to the total number of pixels, which indicates the total percentage of classification [51]. The UA is the ratio of the total number of pixels that are correctly assigned to a class to the total number of pixels from the entire image that the classifier places into this class, while PA refers to the ratio of the number of pixels of a single class correctly classified by the classifier into the total number of pixels of this class. The KC measures the agreement between the classified and reference samples [52,53].

\subsubsection{Time Series Rapid Mapping by Training Sample Migration}

Despite great progress in land cover mapping and urban green space mapping, insufficient and unreliable reference samples to train machine learning algorithms directly impact the accuracy of the supervised classification [54]. It is important to collect consistent, timely, and accurate training samples in order to produce up-to-date green space mapping for urban planning [17]. Therefore, it is necessary to develop a method to migrate the training samples collected in a reference year to other periods for accurate classification. Some studies have employed training samples derived from previous land cover maps for the classification of current images [55]. Inspired by some studies mapping global or immense land cover for any target year using migrated training samples in GEE [19,27], an efficient and easy to perform method was developed to transfer a combination of (1) ground truth samples collected in a reference year and (2) high-quality training samples generated for the target year in order to produce up-to-date UGS maps for any given year without the manual collection of training samples.

In the proposed method (Figure 3), in order to ensure that the migrated training samples are of high quality, both an accurate green space map and a high-quality set of training samples are integrated. In previous studies, since only training samples from reference land cover products were collected [56], the quality of the training samples could be affected by the inherent classification errors from the previous map. Additionally, the use of only ground truth training samples from the reference year leads to the drawback 
that the ground truth dataset for a reference year is unbalanced due to constant land cover change, leading to unreliable classification results [17].

The procedure for the method of migration of training samples includes, (1) generating candidate training samples from a reference green space map, (2) calculating the spectral angle distance (SAD) and Euclidean distance (ED) between reference spectra and target spectra, and (3) eliminating pixels with a high probability of changes in images from different years by a given threshold, thus providing unchanged and high-quality training samples for the target year. SAD and ED measure the change in spectra between the reference year and target year in both the candidate and ground truth training samples, which are considered to be the best magnitude and similarity measures for the detection of bi-temporal changes [57].

By measuring the direction of change between the two temporal vectors, SAD normalizes for the influence of shading and can accentuate the target spectral shape characteristics [58]:

$$
\begin{gathered}
\theta=\cos ^{-1} \frac{\sum_{i=1}^{i=N} X_{i}^{t_{1}} X_{i}^{t_{2}}}{\sqrt{\sum_{i=1}^{i=N}\left(X_{i}^{t_{1}}\right)^{2} \sum_{i=1}^{i=N}\left(X_{i}^{t_{2}}\right)^{2}}} \\
S A D=\cos (\theta)
\end{gathered}
$$

where $\theta$ is the spectral angle expressed in radians $(0-\pi)$. The variable $i$ corresponds to the spectral band and ranges from one to the number of bands $(N)$. Here, $i$ represents 14 spectral features for the Sentinel- 2 images. $X_{i}^{t_{1}}$ is the reference spectra when both the candidate and ground truth training samples are collected at time $t_{1}$, and $X_{i}^{t_{2}}$ is the target spectra to be measured at time $t_{2}$.

ED is the Euclidean distance between two temporal spectra $\left(X_{i}^{t_{1}}\right.$ and $\left.X_{i}^{t_{2}}\right)$, expressed as the square root of the band-wise sum of the squares of the differences [19]:

$$
E D=\sqrt{\sum_{i=1}^{N}\left(X_{i}^{t_{1}}-X_{i=1}^{t_{2}}\right)^{2}}
$$

In this study, to determine a reasonable threshold for judging the spectral change, a tail-and-error procedure was conducted. In addition, the remaining training sample pixels were detected by $\mathrm{SAD}, \mathrm{ED}$, and an intersection criterion $(\mathrm{SAD} \geq 0.88$ and $\mathrm{ED} \leq 0.2)$.

\subsubsection{Dynamic Evaluation of UGS by Quality Indicators}

Recent studies have highlighted the importance of perceived quality in addition to the amount of green space when examining the beneficial effects of green space $[59,60]$. Some studies have assessed greenness using the Normalized Difference Vegetation Index (NDVI), which shows the most current urban green space density and distribution; studies that rely solely on NDVI reduce the complexity of nature to mere "greenness", which may misclassify exposures and lead to limited assessment results [61]. In addition to greenness, the quality of green spaces is intricately linked to their configurational aspects, as elongated and well-distributed green space patches attract and benefit residents better [62] Accordingly, we used six UGS quality indicators (Table 1) to assess the quality of UGS distribution. Among these, four metrics were used, including UGS patch density (PD), mean area of UGS (AREA_MN), and aggregation index (AI), which describe the objective quality of UGSs, since small, fragmented, and simple-shaped patches provide poorer service than large, contiguous, and complex-shaped patches. The four metrics of green spaces were computed from 2016 to 2020 from the respective green space maps and assessed for each district. Furthermore, two indicators, the urban green index (UGI) and Shannon's diversity index (SHDI), represent the vegetation coverage and green patch diversity around pixels (in $300 \mathrm{~m}$ liner distance). The surrounding greening quality assessment based on a moving window method was to build a circular window with a radius of $300 \mathrm{~m}$, calculate the ratio of the number of green pixels to the total number of pixels in the window, define 
the ratio as the UGI of the central pixel of the window, and traverse each pixel of the study area to obtain the UGI within the whole area.

Table 1. Description of urban green space landscape metrics used in the study.

\begin{tabular}{|c|c|c|c|}
\hline Metrics & Formula & Description & Units and Range \\
\hline PD & $P D=\frac{n}{A_{D}} \times 10^{6}$ & $\begin{array}{l}\text { where } n \text { is the number of UGS patches in the } \\
\text { district, and } A_{D} \text { is the area of the district }\end{array}$ & number per $\mathrm{km}^{2} ; \mathrm{PD} \geq 0$ \\
\hline AREA_MN & Area $_{M N}=\frac{A_{u G S}}{n} \times 10^{-4}$ & $\begin{array}{l}\text { where } A_{U G S} \text { is the area of UGS (in } \mathrm{m}^{2} \text { ) in the } \\
\text { district, and } n \text { is the number of UGS patches } \\
\text { in the district }\end{array}$ & ha; Area_MN $\geq 0$ \\
\hline FRAC_AM & $\begin{array}{c}F R A C_{A M}= \\
\sum_{i=1}^{i=n}\left(\frac{a_{i}}{A_{U G S}}\right) \cdot\left(\frac{2 \ln 0.25 p_{i}}{\ln a_{i}}\right)\end{array}$ & $\begin{array}{l}\text { where } a_{i} \text { and } p_{i} \text { denote the area }\left(\text { in } \mathrm{m}^{2} \text { ) and }\right. \\
\text { perimeter (in } \mathrm{m} \text { ) of a UGS patch } i, n \text { is the } \\
\text { number of UGS patches in the district, and } \\
A_{U G S} \text { is the area (in } \mathrm{m}^{2} \text { ) of UGS in the district }\end{array}$ & $\begin{array}{l}1 \leq \text { FRAC_AM } \leq 2 \\
\text { FRAC_AM }=1 \text { if the patches are } \\
\text { square-shaped } \\
\text { FRAC_AM }=2 \text { if the patches are } \\
\text { highly convoluted }\end{array}$ \\
\hline $\mathrm{AI}$ & $A I=\left(\frac{J}{\max J}\right) \times 100$ & $\begin{array}{l}\text { where } J \text { is the number of connections } \\
\text { between pixels of UGS patches in the district } \\
\text { based on the single-count method, and max } J \\
\text { is the maximum possible value of } J\end{array}$ & $\begin{array}{l}\text { Percent; } 0 \leq \mathrm{AI} \leq 100 \\
\mathrm{AI}=0 \text { if the patches are } \\
\text { maximally } \\
\text { disaggregated } \\
\mathrm{AI}=100 \text { if the patches are } \\
\text { maximally } \\
\text { compact }\end{array}$ \\
\hline UGI & $U G I=\frac{N_{U G S}}{N_{T N N}} \times 100 \%$ & $\begin{array}{l}\text { the } U G I \text { of a pixel is defined as the ratio of } \\
\text { the number of green pixels near the pixel (in } \\
300 \text { m linear distance) }\left(N_{U G S}\right) \text { to the number } \\
\text { of all pixels nearby }\left(N_{T N N}\right)\end{array}$ & $\begin{array}{l}\text { Represents the vegetation } \\
\text { coverage around the pixel }\end{array}$ \\
\hline SHDI & $S H D I=-\sum_{i=1}^{m} p i \times \ln (p i)$ & $\begin{array}{l}\text { where } m \text { represents the total number of } \\
\text { vegetation-type patches nearby (in } 300 \mathrm{~m} \\
\text { liner distance), and } p_{i} \text { represents the area } \\
\text { proportion of the patch } i\end{array}$ & $\begin{array}{l}\text { Represents the diversity around } \\
\text { the pixel }\end{array}$ \\
\hline
\end{tabular}

An additional two indicators, the area of UGS (AUGS, $\mathrm{km}^{2}$ ) and the percentage of the UGS area (PUGS, \%) assess the quantity of UGS in each district. The latter indicator is defined as the ratio of the AUGS to the total area $\left(\mathrm{km}^{2}\right)$ of the district and ranges from 0 to 100.

\section{Results}

\subsection{UGS Classification Maps and the Accuracy Assessment Results}

In this section, the classification accuracy of the different numbers of input features are compared using the RF classifier for the classification of different vegetation types; then, we analyze the importance of different input features, and the best combinations of input features are utilized to produce the urban green space map. Finally, the accuracy of the migration of reference samples for automatic green space mapping is discussed.

\subsubsection{Comparative Classification Accuracy of Different Input Feature Configurations}

In this study, a total of 39 types of features were calculated, including 14 spectral features, 5 vegetation indices, 18 texture features, and the elevation band and slope band. One of the significant advantages of the RF classifier is that the importance of each feature can be evaluated. This is beneficial when many input features are incorporated into the classifier. In GEE, it recently became possible to output the analysis of the feature importance. Based on the training samples collected from all six districts, we evaluated the importance of each feature using the RF algorithm. Figure 4 demonstrates the importance factor of each feature. 


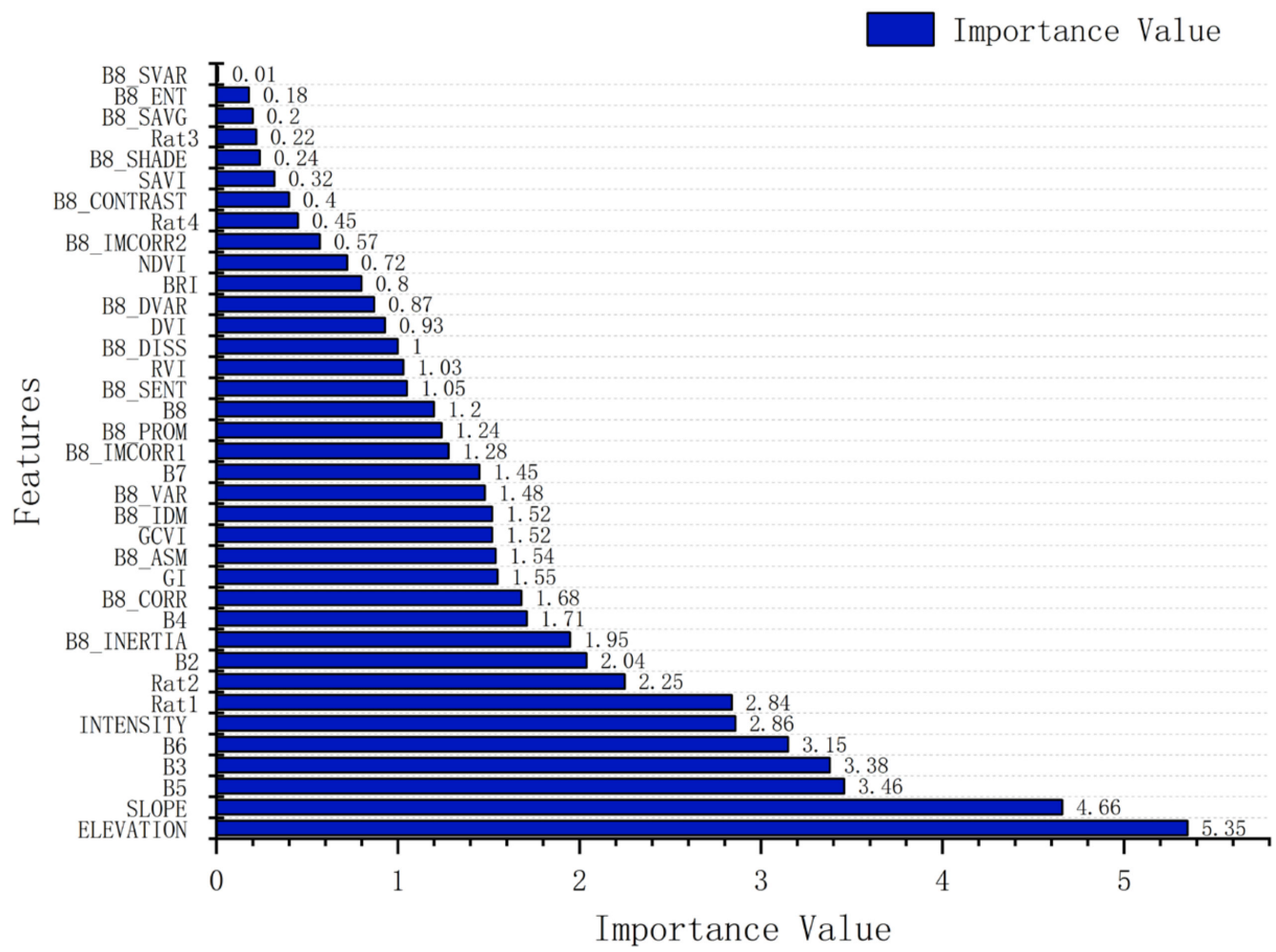

Figure 4. Importance value of image features.

Overall, spectral features show higher importance than other kinds of features except for elevation features and the slope band. The most important spectral features are the Red Edge band 1 and Green band, followed by the Red Edge band 2 and Intensity band. Textural features, such as Inertia and Correlation, also show great importance for achieving higher accuracies. GI and GCVI are important vegetation indices features for the classification of different vegetation types.

We compared the classification accuracy of different configurations of input features, as validated by the test samples, which are listed in Table 2 .

Table 2. Accuracy assessment of different input features configurations.

\begin{tabular}{ccc}
\hline \multirow{2}{*}{ Numbers of Input Features } & \multicolumn{2}{c}{ RF Classifier } \\
\cline { 2 - 3 } & OA(\%) & KC(\%) \\
\hline 12 & 93.06 & 88.98 \\
20 & 96.60 & 94.40 \\
25 & 94.49 & 94.16 \\
39 & 92.60 & 88.00 \\
\hline
\end{tabular}

That shows that with an increase in the number of features, the classification accuracy is continuously improved. When the number of features exceeds 20 , the classification accuracy is reduced. Too many feature variables resulted in a low calculation efficiency and a low classification accuracy. The 20 features with the highest importance values were selected as input feature combinations for classification. With the exception of the number of feature variables, the number of trees is the most critical parameter for RF-based classification. In our study, an automatic suitable parameter selection based on a statistical accuracy assessment for the number of trees was edited on the GEE JavaScript editor. 


\subsubsection{Accuracy Assessment for Differentiating Vegetation Type Using Different} Supervised Classifiers

In the proposed method (Figure 3), the number of migrated training samples generated from the reference UGS map is variable, and a low quantity of training data affects the performance of classification. The GEE environment integrates several different classifiers. Non-parametric algorithms, such as CART, non-linear SVM, and RF, were implemented to produce the UGS map for 2016. The performance of the classifiers with the best combination of parameters was analyzed and compared using a confusion matrix, and the corresponding user accuracy (UA), producer accuracy (PA), and overall accuracy (OA), along with the kappa coefficient (KC), were estimated (Table 3).

Table 3. Accuracy assessment of different machine learning classifiers.

\begin{tabular}{ccccccc}
\hline Classifier & \multicolumn{2}{c}{ RF } & \multicolumn{2}{c}{ SVM } & \multicolumn{2}{c}{ CART } \\
\hline Accuracy & UA(\%) & PA(\%) & UA(\%) & PA(\%) & UA(\%) & PA(\%) \\
Urban trees & 90.40 & 98.80 & 92.10 & 95.30 & 91.00 & 94.20 \\
Low plants & 95.70 & 71.00 & 85.70 & 77.40 & 85.20 & 74.20 \\
Forest & 100 & 100 & 100 & 100 & 98.00 & 100 \\
OA (\%) & & 94.00 & \multicolumn{2}{c}{93.04} & \multicolumn{2}{c}{92.20} \\
KC (\%) & \multicolumn{2}{c}{89.90} & \multicolumn{2}{c}{89.00} & \multicolumn{2}{c}{87.00} \\
\hline
\end{tabular}

The best accuracy values were achieved when performing classification with RF, obtaining an OA of $94.00 \%$ and a KC of $89.90 \%$. SVM produced results with slightly lower accuracy than that of RF, and the estimated $\mathrm{KC}$ and OA were $93.04 \%$ and $89.00 \%$, respectively. CART provided a lower accuracy than that of RF and SVM.

We also analyzed the influence of different numbers of training samples on the classification results, and the results of the different classifiers are shown in Figure 5.

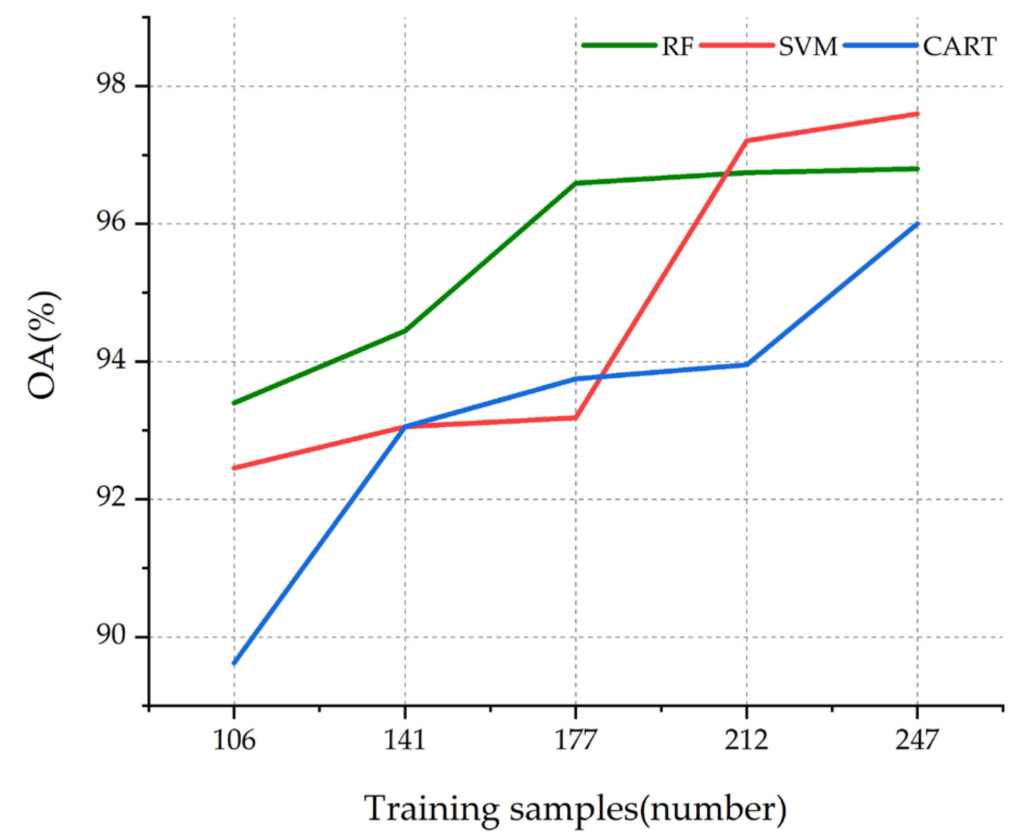

Figure 5. Accuracy of the different number of training samples for three machine learning classifiers.

As seen in Figure 5, compared with other classifiers, the RF classifier is insensitive to the number of training samples, and its classification accuracy is stable. When the number of training samples was 212 and 247, the OA of the SVM classifier was higher than that of RF, but the change in the number of training samples had a significant impact on the classification accuracy of SVM. The OA of the CART classifier was lower than that of RF and SVM for different training sample sizes. Since the training sample migration method adopted in this 
paper causes the number of training samples to fluctuate annually, in order to obtain a stable classification accuracy, this paper uses an RF classifier instead of an SVM classifier.

\subsubsection{Time Series Classification Result Using Migrated Training Samples}

Figure 6 shows the UGS classification result in the study area using the 20 optimal features with the RF algorithm. Since the RF classifier has shown a high accuracy and execution speed, it can be beneficial to produce up-to-date maps of green spaces in city areas. An automatic method was proposed in Section 2.2.3. to obtain a green infrastructure map for any target year by migrating training samples. In our study, 2016 was selected as the reference year; both the 2016 ground truth training samples and numerous unchanged candidate training samples randomly generated from the 2016 UGS map were considered as reference training samples for the target years 2017-2020. The unchanged training samples were detected by SAD and ED.

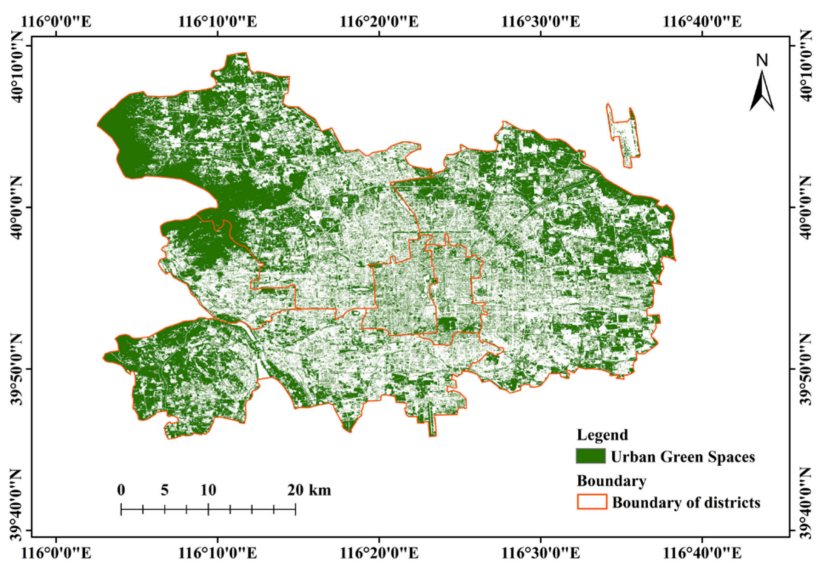

(a) 2016

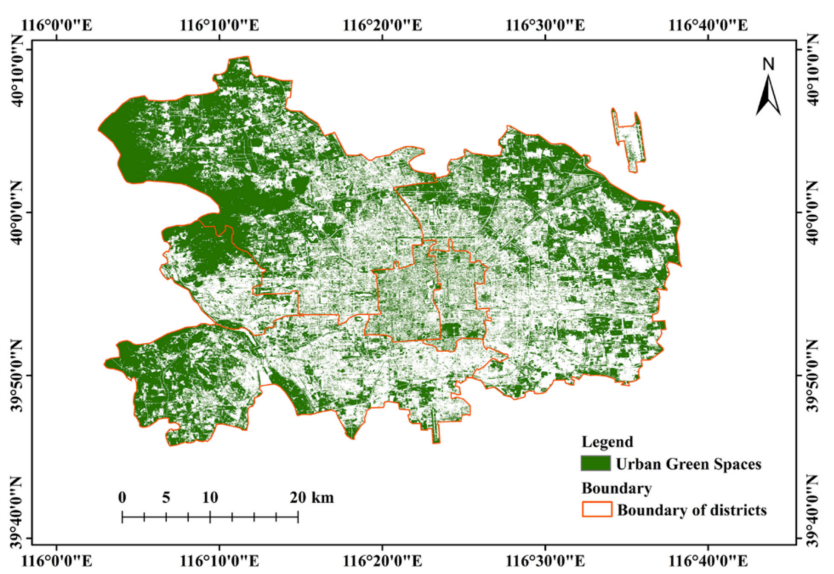

(c) 2017

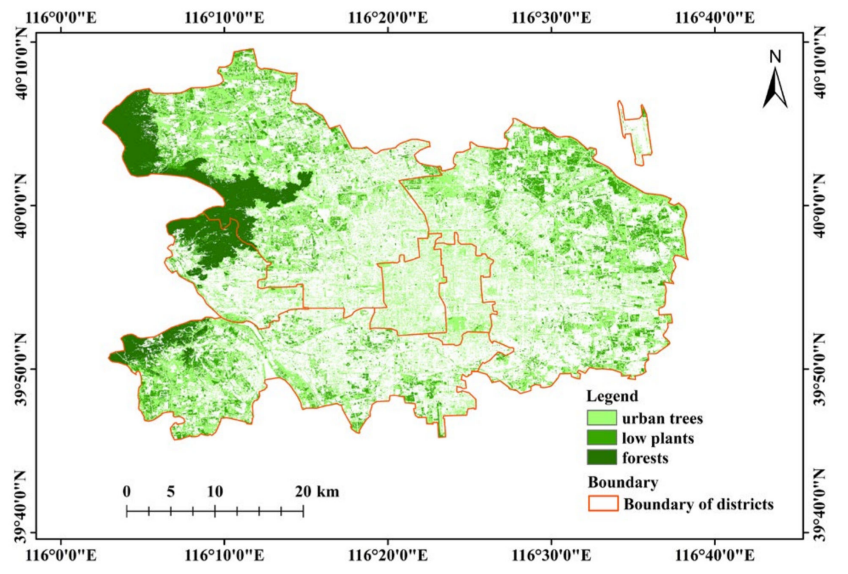

(b) 2016

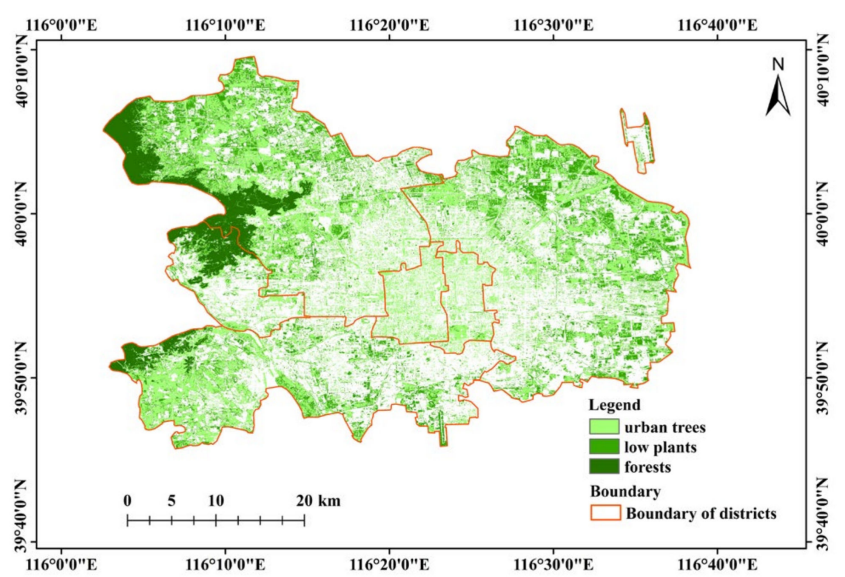

(d) 2017

Figure 6. Cont. 


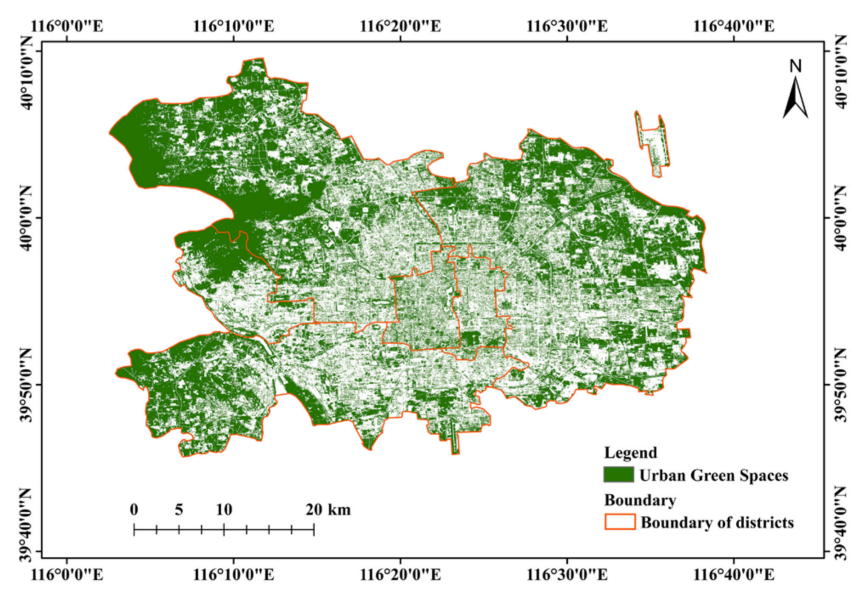

(e) 2018

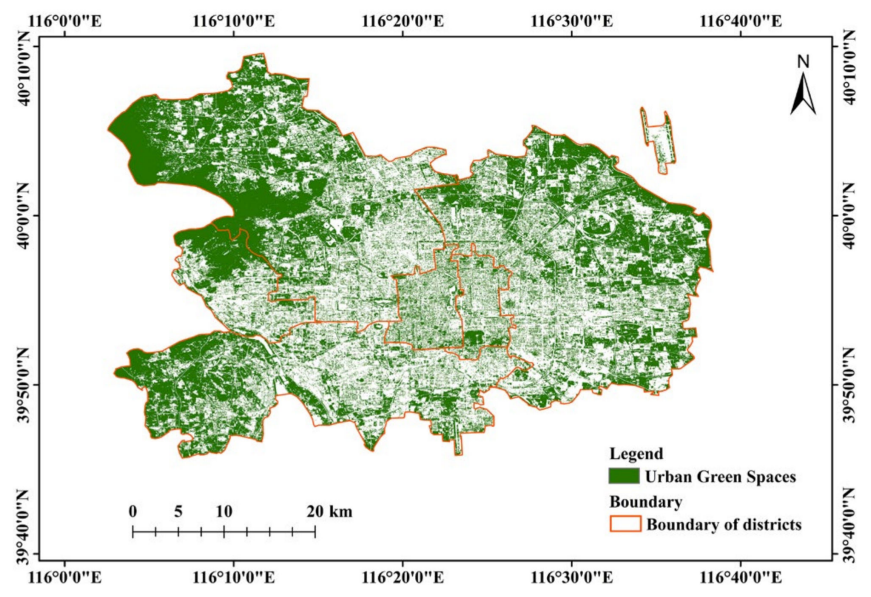

(g) 2019

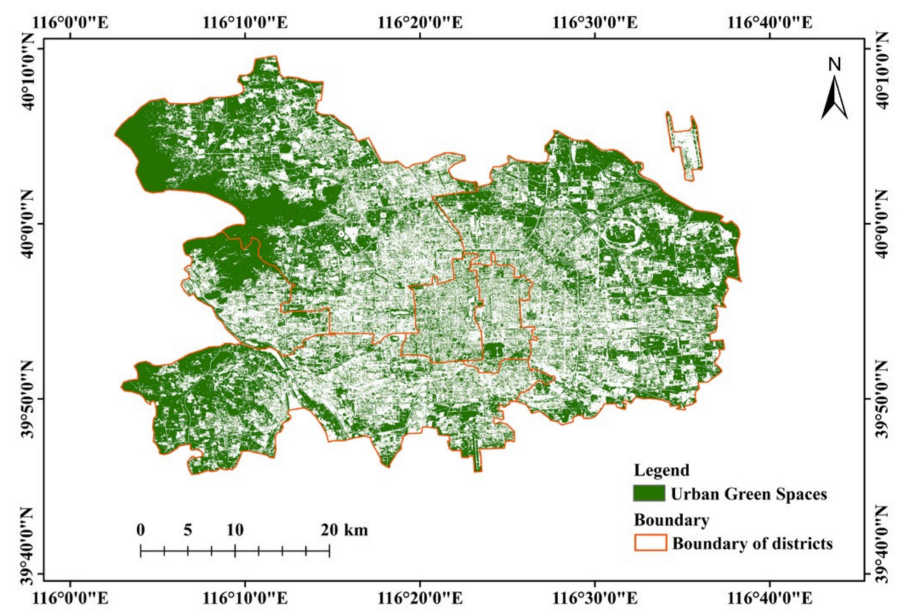

(i) 2020

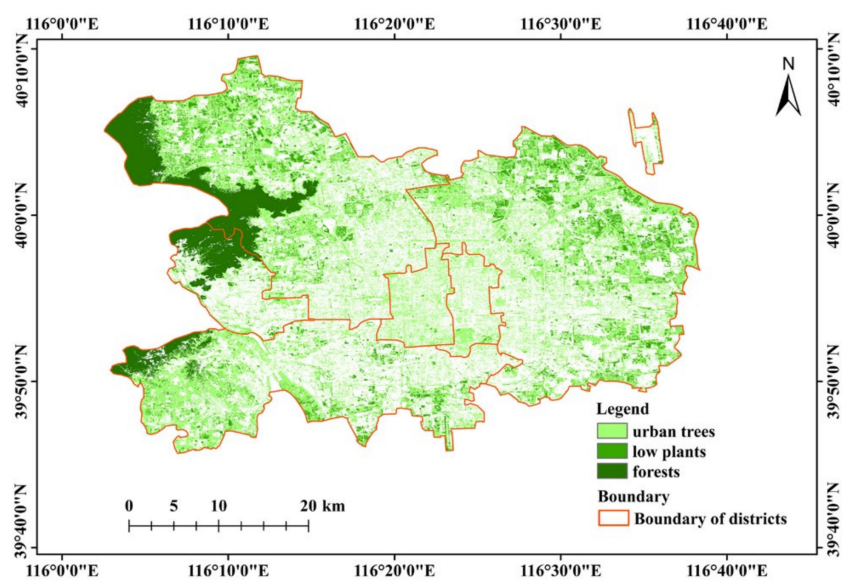

(f) 2018

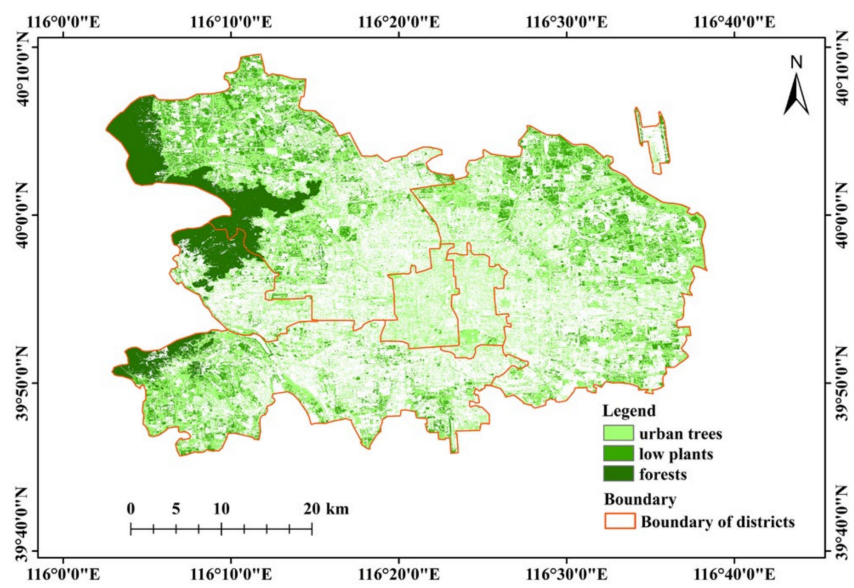

(h) 2019

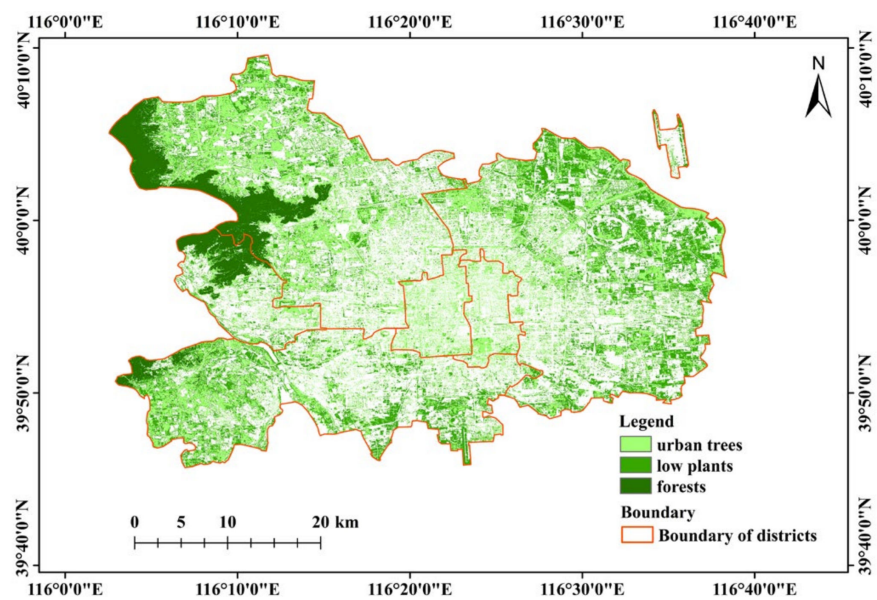

(j) 2020

Figure 6. Extraction result (a,c,e,g,i) and classification result $(\mathbf{b}, \mathbf{d}, \mathbf{f}, \mathbf{h}, \mathbf{j})$ of UGS in the center of Beijing from 2016 to 2020. 
The average OA and KC from 2016 to 2020 of the produced green space maps using migrated training samples and the RF classifier were $96.47 \%$ and $94.25 \%$, respectively, suggesting the high efficiency of the proposed workflow for automatic UGS mapping (Table 4).

Table 4. Accuracy of annual UGS maps from 2016 to 2020.

\begin{tabular}{ccccccccccc}
\hline Year & \multicolumn{2}{c}{$\mathbf{2 0 1 6}$} & \multicolumn{2}{c}{$\mathbf{2 0 1 7}$} & \multicolumn{2}{c}{$\mathbf{2 0 1 8}$} & \multicolumn{2}{c}{$\mathbf{2 0 1 9}$} & \multicolumn{2}{c}{$\mathbf{2 0 2 0}$} \\
\hline $\begin{array}{c}\text { Accuracy } \\
\text { Urban }\end{array}$ & $\mathrm{UA}(\%)$ & $\mathrm{PA}(\%)$ & $\mathrm{UA}(\%)$ & $\mathrm{PA}(\%)$ & $\mathrm{UA}(\%)$ & $\mathrm{PA}(\%)$ & $\mathrm{UA}(\%)$ & $\mathrm{PA}(\%)$ & $\mathrm{UA}(\%)$ & $\mathrm{PA}(\%)$ \\
trees & 93.18 & 97.62 & 97.70 & 96.59 & 95.73 & 94.12 & 97.94 & 98.96 & 97.73 & 94.51 \\
Low & & & & & & & & & & \\
plants & 97.50 & 95.12 & 94.28 & 91.67 & 92.31 & 94.74 & 100 & 95.24 & 85.71 & 93.75 \\
Forest & 100 & 98.04 & 100 & 96.08 & 92.42 & 63.85 & 98.31 & 100 & 100 & 100 \\
OA(\%) & 97.01 & 96.53 & 94.40 & 98.47 & 95.93 \\
KC(\%) & 95.50 & 94.39 & 90.48 & 97.55 & 93.31 \\
\hline
\end{tabular}

\subsection{Temporal Changes in the Spatial Distribution of UGS from 2016 to 2020}

By comparing the UGS maps for 2016 to those for 2020 (Figure 6), we found that the green spaces in the center of Beijing had a similar distribution. Furthermore, to qualitatively assess the temporal changes in UGSs in the study area from 2016 to 2020, the four landscape pattern metrics derived from 2016 to 2020 are listed in Table 4.

The results (Table 5) show that the UGS in the center of Beijing increased by $48.62 \mathrm{~km}^{2}$ from 2016 to 2020. Subsequently, the PUGS in the city increased by $7.24 \%$ during this period. Furthermore, the green space patch density (PD) values decreased by $5.88 \%$, and the mean area of the UGS patches (Area_MN) increased by 0.03 ha, indicating that the fragmentation of the UGS was reduced in this period. The changes in the aggregation index (AI) and area-weighted mean fractal dimension index (FRAC_AM) values suggest that the UGS was relatively compact and regular during this period. Thus, the green space in the center of Beijing increased and became more integrated and compact during 2016-2020.

Table 5. Temporal changes in four types of urban green space distribution indicators.

\begin{tabular}{ccccccc}
\hline \multirow{2}{*}{ Year } & \multicolumn{7}{c}{ UGS Distribution Indicators } \\
\cline { 2 - 7 } & AUGS $\left(\mathbf{k m}^{\mathbf{2}}\right)$ & PUGS (\%) & Area_MN & FRAC_AM & AI & PD \\
\hline 2016 & 671.59 & 48.48 & 0.24 & 1.29 & 77.117 & 193.99 \\
2020 & 720.21 & 51.99 & 0.27 & 1.27 & 77.82 & 182.58 \\
\hline
\end{tabular}

The AUGS and PUGS in Chaoyang, Fengtai, Shijingshan, and Xicheng increased from 2016 to 2020 (Table 6). The UGS in Chaoyang District became larger and more aggregated; at the same time, vegetation fragmentation in Chaoyang District was reduced during this period. Haidian District had the largest AUGS and PUGS, and fragmentation was reduced from 2016 to 2020. The PUGS and AUGS of Dongcheng and Xicheng Districts were relatively small, and the mean area of the UGS patches increased during this period. 
Table 6. Descriptive statistics of urban green space distribution indicators for six districts in 2016 and 2020.

\begin{tabular}{|c|c|c|c|c|c|c|c|c|c|c|c|c|}
\hline \multirow{3}{*}{ District } & \multicolumn{12}{|c|}{ UGS Distribution Indicators } \\
\hline & \multicolumn{2}{|c|}{ AUGS $\left(\mathbf{k m}^{2}\right)$} & \multicolumn{2}{|c|}{ PUGS (\%) } & \multicolumn{2}{|c|}{ Area_MN } & \multicolumn{2}{|c|}{ FRAC_AM } & \multicolumn{2}{|c|}{ AI } & \multicolumn{2}{|c|}{ PD } \\
\hline & 2016 & 2020 & 2016 & 2020 & 2016 & 2020 & 2016 & 2020 & 2016 & 2020 & 2016 & 2020 \\
\hline Chaoyang & 207.30 & 234.13 & 44.03 & 49.73 & 0.22 & 0.26 & 1.28 & 1.27 & 74.56 & 76.43 & 198.64 & 184.72 \\
\hline Dongcheng & 15.88 & 15.60 & 37.96 & 37.29 & 0.20 & 0.20 & 1.27 & 1.26 & 73.45 & 72.17 & 187.75 & 176.49 \\
\hline Fengtai & 136.23 & 156.59 & 44.52 & 51.17 & 0.23 & 0.26 & 1.28 & 1.27 & 77.11 & 75.84 & 190.75 & 189.26 \\
\hline Haidian & 251.18 & 247.28 & 58.31 & 57.4 & 0.29 & 0.31 & 1.30 & 1.28 & 79.20 & 80.81 & 199.37 & 179.71 \\
\hline Shijingshan & 41.8 & 46.33 & 48.75 & 54.03 & 0.29 & 0.27 & 1.28 & 1.27 & 81.29 & 80.51 & 168.48 & 195.62 \\
\hline Xicheng & 19.2 & 20.28 & 37.86 & 40.00 & 0.20 & 0.25 & 1.28 & 1.28 & 72.05 & 71.62 & 185.78 & 146.65 \\
\hline
\end{tabular}

3.3. Annual Dynamic Evaluation of the Quality of UGS by Using UGI and SHDI at Pixel Level 3.3.1. Urban Green Index (UGI)

The UGI index represents the vegetation coverage rate of $300 \mathrm{~m}$ around the central pixel. In order to distinguish the difference in the pixel's UGI values, by referring to the principle of equal spacing and combining it with the samples collected from Google Earth high-resolution images, the UGI values from the center of Beijing are divided into the following six grades: lowest $(0.00-0.17)$, low $(0.17-0.34)$, medium $(0.34-0.50)$, relatively high (0.50-0.67), high (0.67-0.84), and highest (0.84-1.00). The proportion of the six UGI levels in the six districts by spatial statistics from 2016 to 2020 is shown in Figure 7.

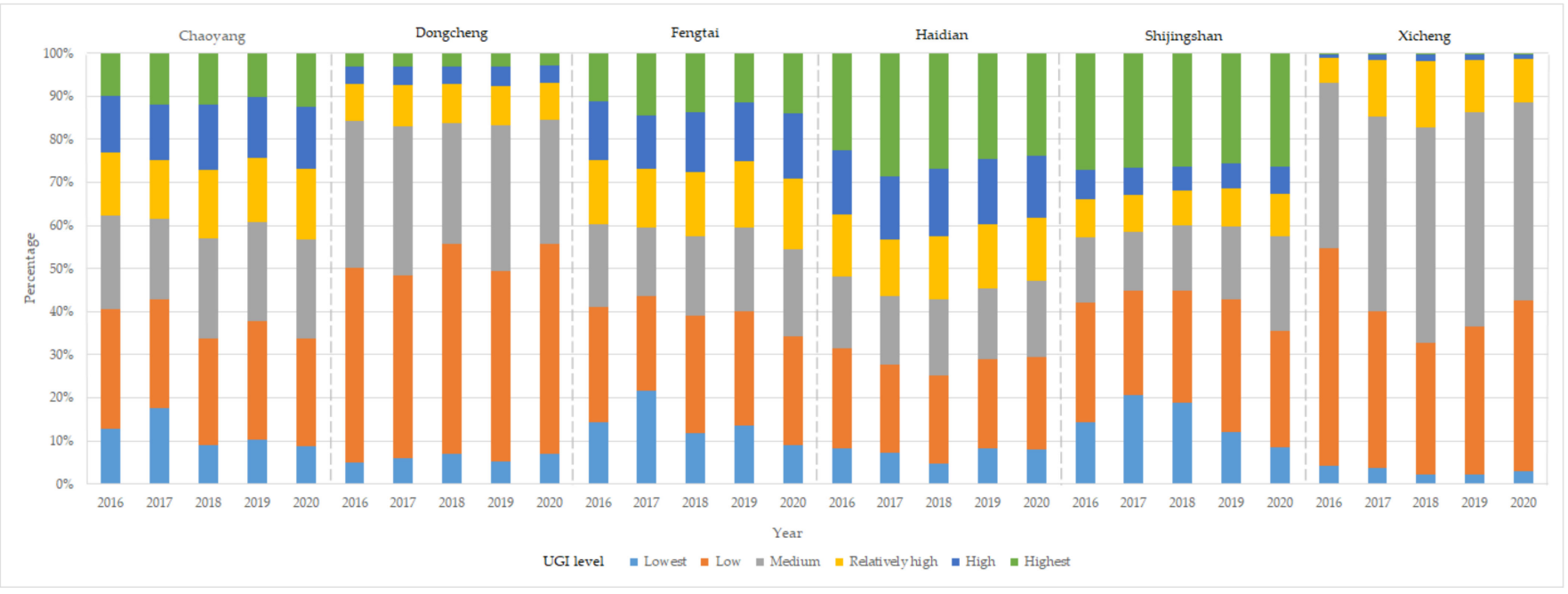

Figure 7. Percentage of different levels of UGI in six districts.

Overall, the average proportion of relatively high and above levels (UGI >0.5) in the six districts was $32.45 \%$ in 2016 and $35.16 \%$ in 2020 , and the maximum was $36.04 \%$ in 2018; the proportion of the high-level UGI in each district remained unchanged, while that of the highest level UGI changed slightly from 2016 to 2020. The area with the highest level of UGI in the Chaoyang, Fengtai, Haidian Districts increased from 2016 to 2017 and then deceased from 2017 to 2020, showing an overall increase. The proportions of the lowest and low levels of UGI fluctuated significantly in all districts from 2016 to 2020; among them, the proportion of the lowest level in Chaoyang District, Fengtai District, Dongcheng District, and Shijingshan District increased and then decreased, and the proportion of the lowest level in Haidian District and Xicheng District decreased and then increased. The proportion of the low-level UGI in Xicheng District decreased from 2016 to 2018 and then increased slightly from 2018 to 2020; overall, the proportion of the low-level UGI in Xicheng District decreased, and the poor vegetation cover was improved from 2016 to 2020. The UGI level in Xicheng and Dongcheng Districts was mainly at the medium level and below medium levels, accounting for more than $80 \%$. Fengtai, Shijingshan, and Chaoyang Districts had 
similar rates of medium and below medium levels, at approximately $60 \%$. The UGI levels in Haidian District were mainly relatively high and above relatively high, accounting for more than 50\%; the lowest percentage was 52\% in 2016 and the highest was 58\% in 2018 .

Figure 8 shows the spatial-temporal changes in UGI in the center of Beijing, with bottle green, green, light green, yellow, orange, and red representing the lowest, low, medium, relatively high, high, and highest levels, respectively. Overall, the periphery of the study area had a higher UGI compared with the center of the study area. High values of UGI were distributed in the north of the study area. Poor vegetation cover levels, including the lowest and low levels, were mainly distributed in the south of the study area. The most significant changes occurred in Fengtai, where the UGI increased from 2016 to 2020.

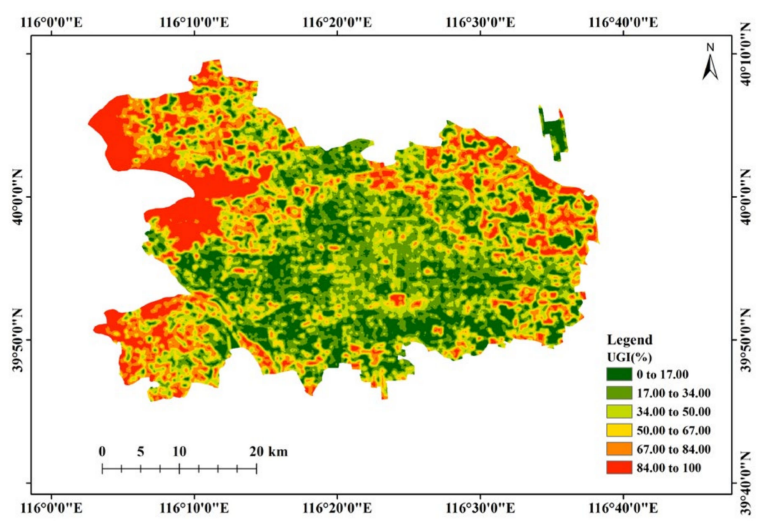

(a) 2016

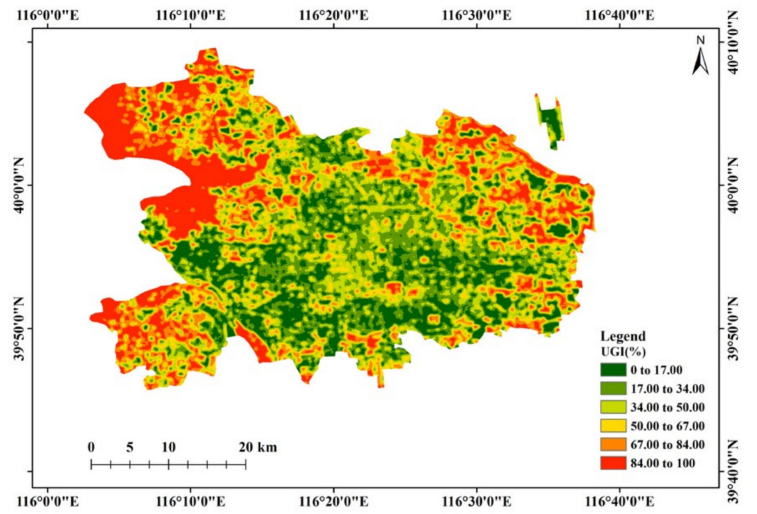

(c) 2018

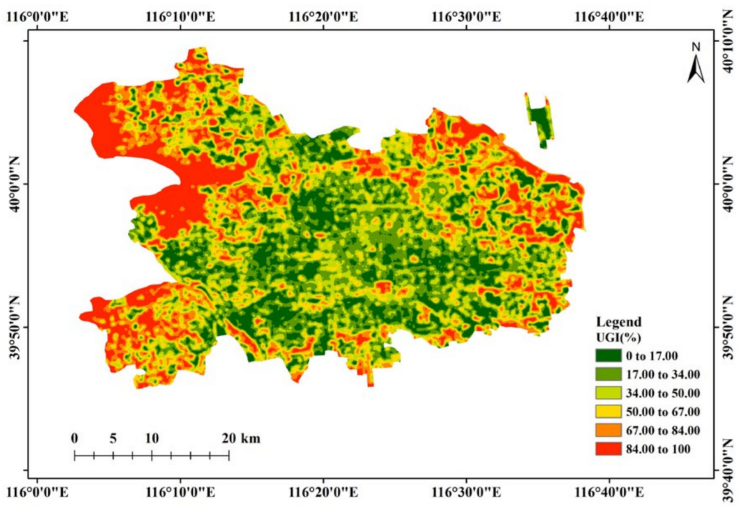

(e) 2020

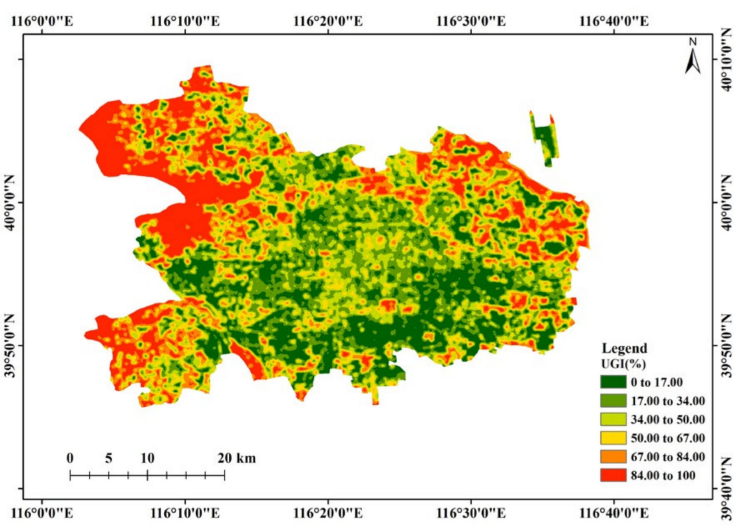

(b) 2017

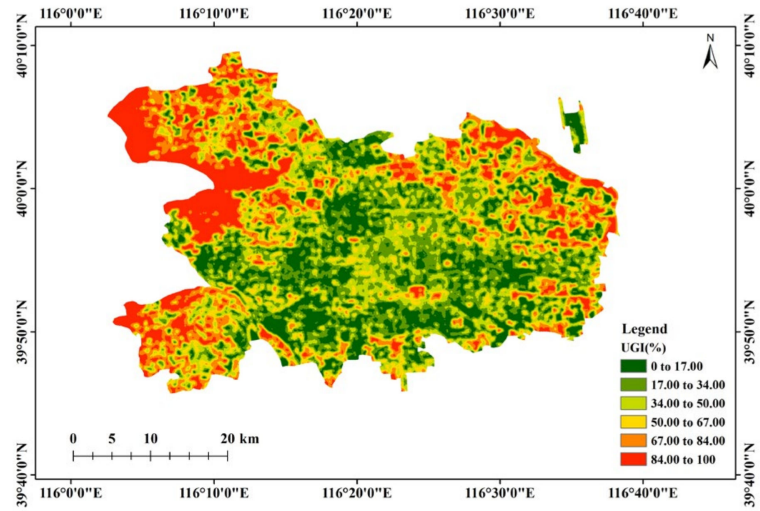

(d) 2019

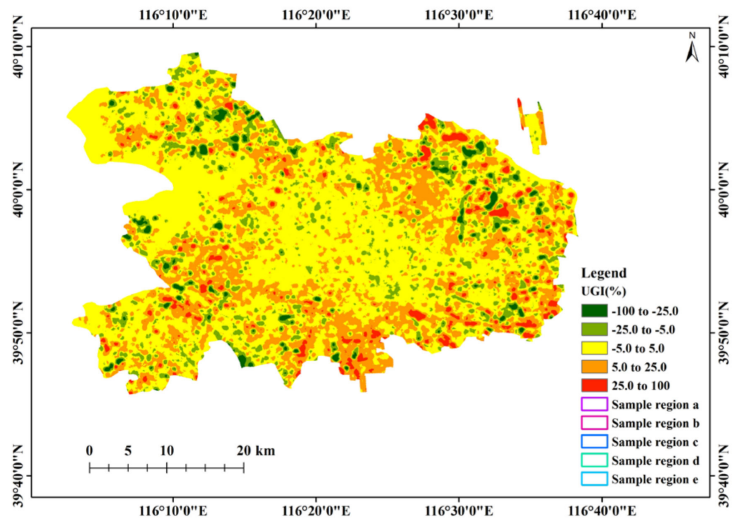

(f) change in UGI from 2016 to 2020

Figure 8. UGI distribution maps of the study area from 2016 to 2020. 
Figure 8f shows the rate of change in UGI values between 2016 and 2020. It shows that intensive vegetation cover changes took place in all directions in the 2020s but were concentrated in the periphery of the study area. A significant increase in the UGI value was observed in the south of the study area, especially in the southeast of Fengtai District and the south of Chaoyang District. The eastern part of the study area experienced both an increase and a decrease in vegetation cover. The change in vegetation coverage in the southwest of the study area was dominated by an increase in vegetation coverage. Figure 9 shows the annual dynamic changes of the UGI in five sampling areas.
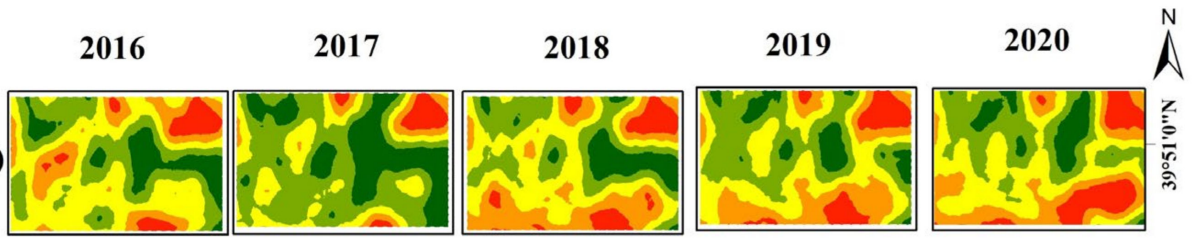

(b)
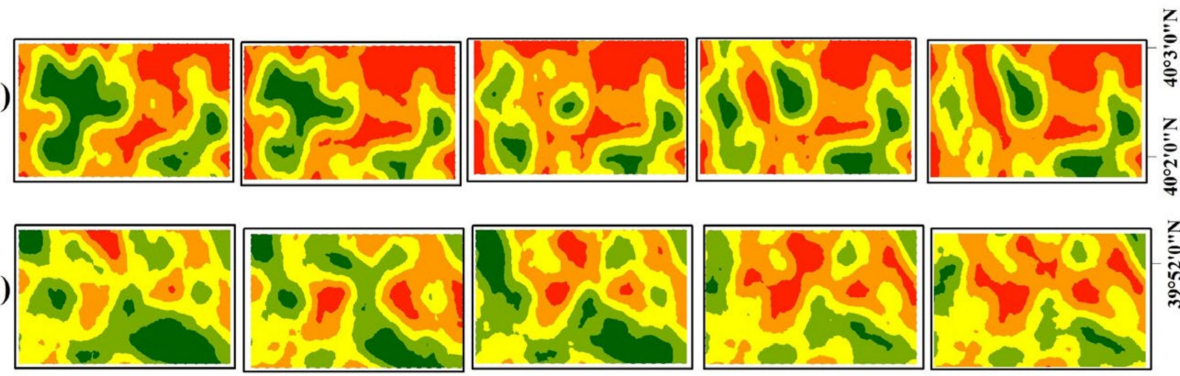

(c)
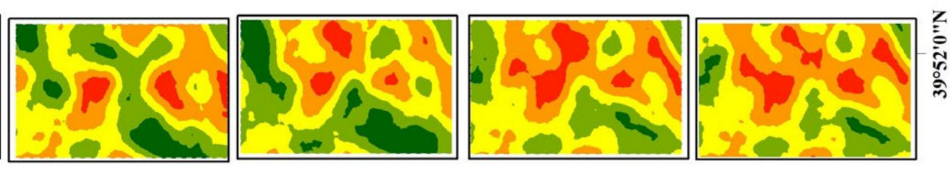

(d)
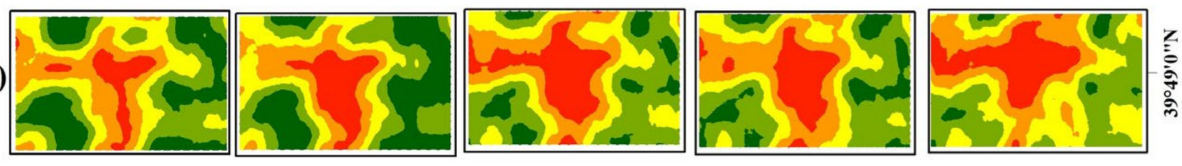

(e)
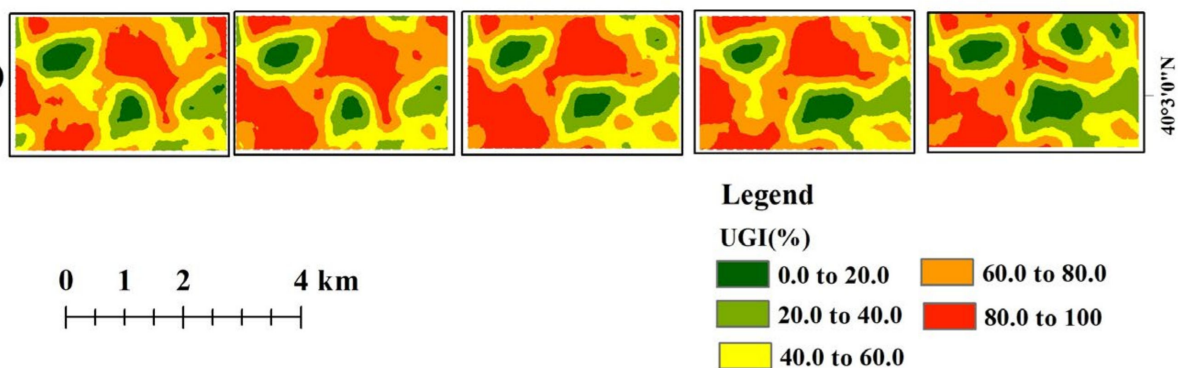

Figure 9. UGI distribution in the five sample regions (a, b, c, d, e in Figure 8f) from 2016 to 2020.

\subsubsection{Shannon's Diversity Index (SHDI)}

The SHDI in the study area is shown in Figure 10. In general, the SHDI values around the study area were higher than those in the center, and the SHDI values in the north of the study area were higher than those in the south.

The most distinguished spatial changes appeared in the south of the study area, particularly in Fengtai District at the southwest corner. The SHDI showed a significant improvement in many places, especially in the center of Beijing; thus, the distribution of Shannon's diversity around the city residents tended to be more even from 2016 to 2020. We calculated the standard deviation of each pixel's SHDI value between 2016 and 2020 (Figure 10f), which showed that the distribution of SHDI had a noticeable fluctuation in the northwest, southwest, and northeast of the study area. This was due to the increase or decrease in low plants areas (Figure 6), which in turn affected the SHDI of these regions. 


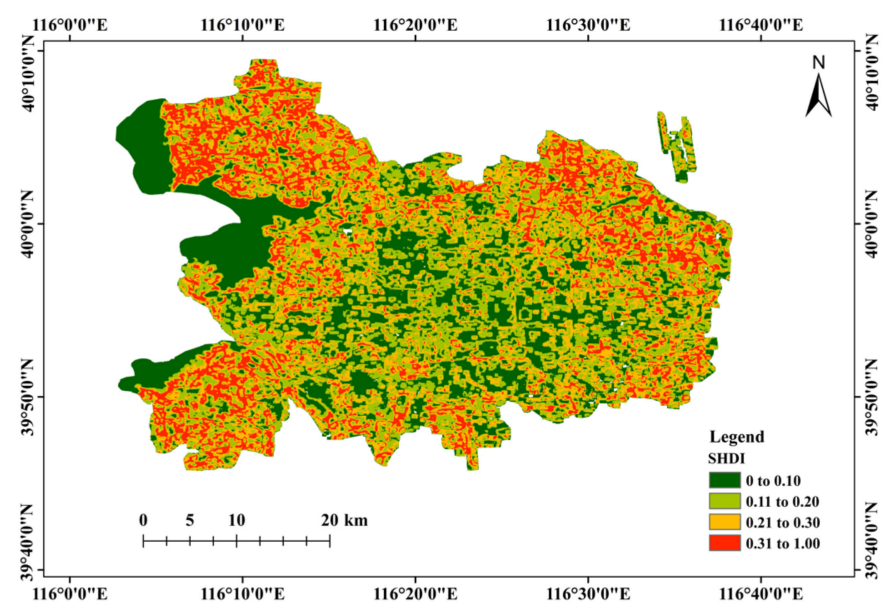

(a) 2016

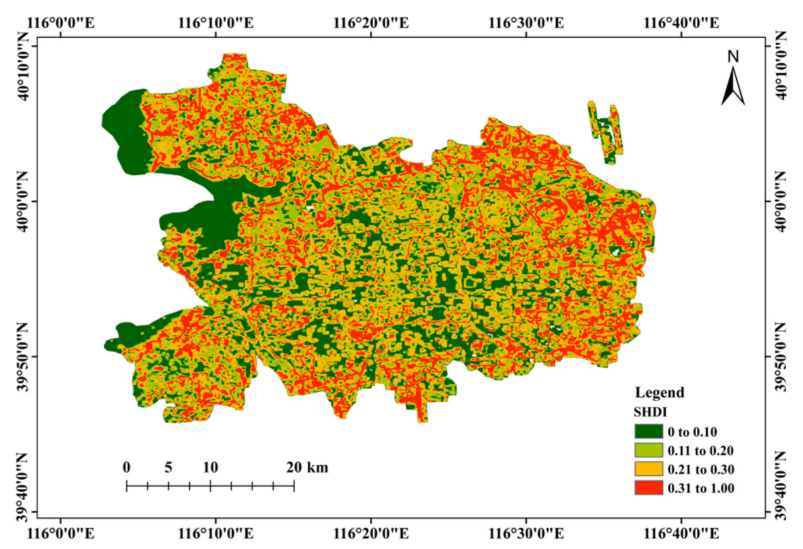

(c) 2018

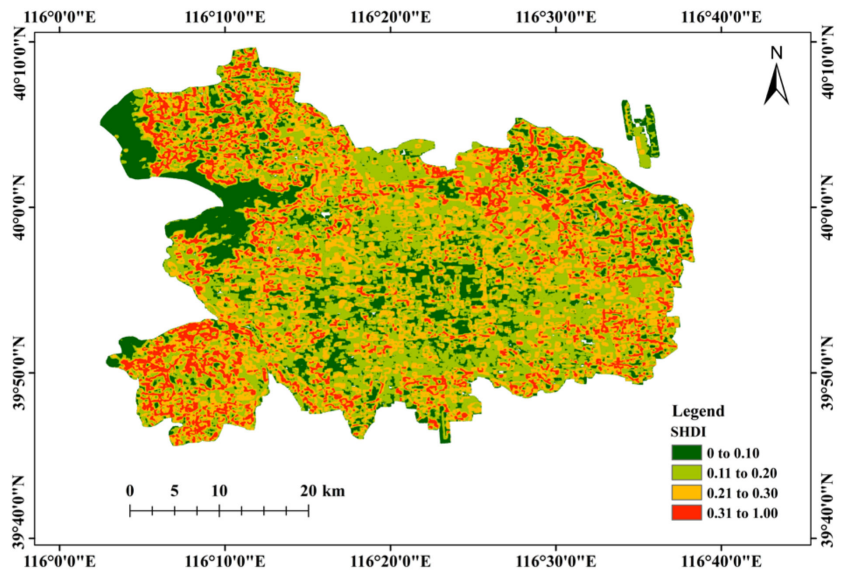

(e) 2020

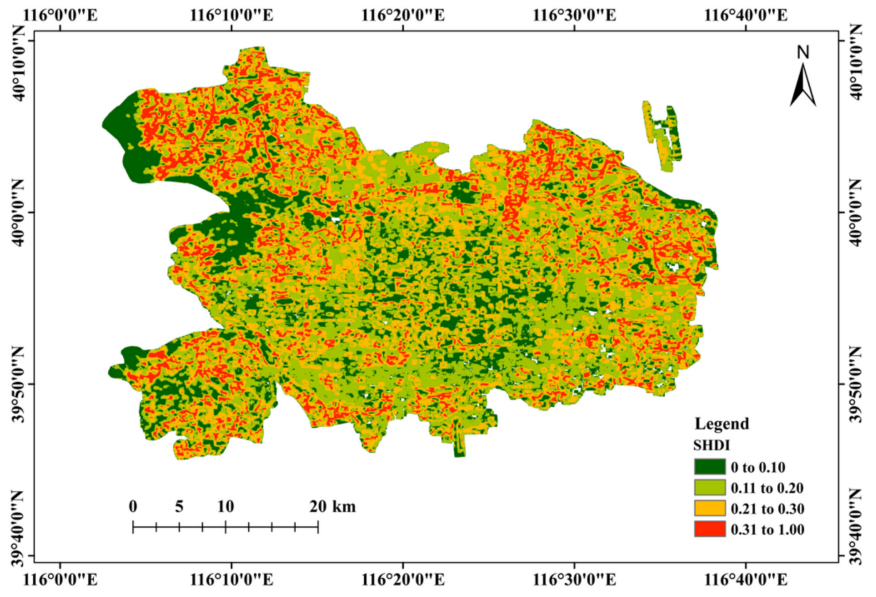

(b) 2017

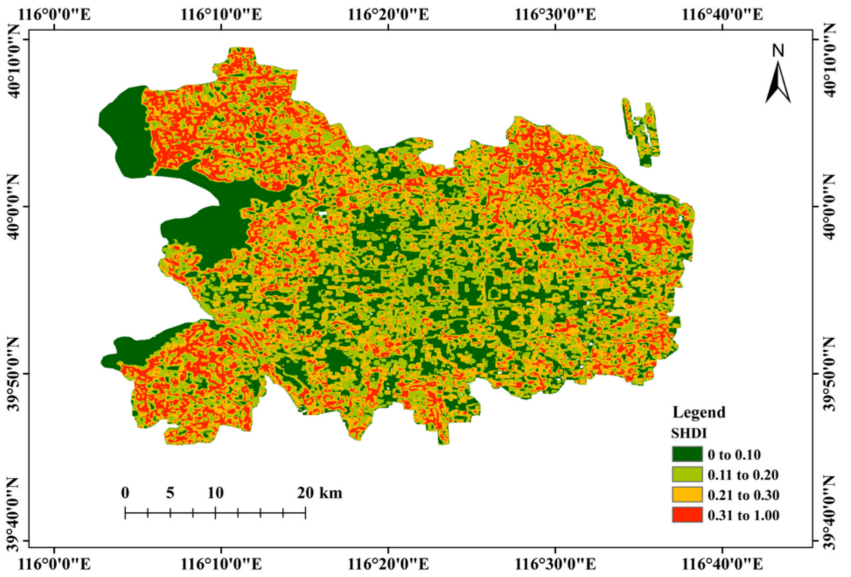

(d) 2019

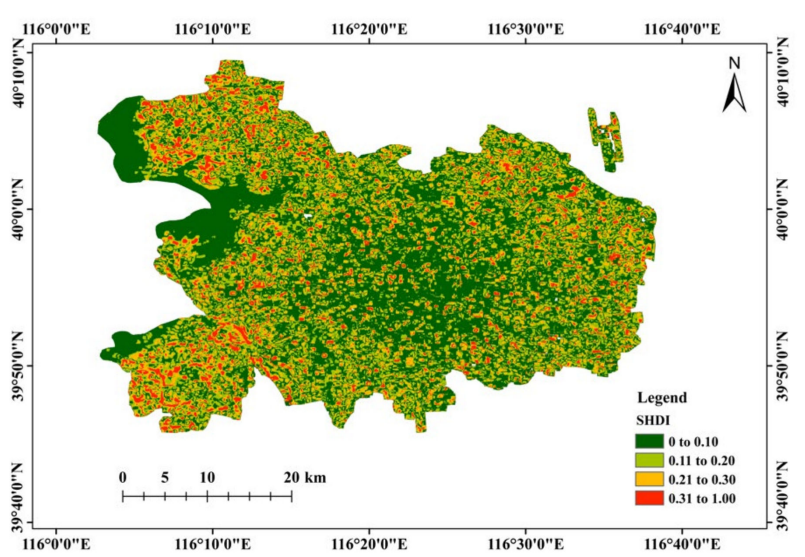

(f) Standard deviation of SHDI

Figure 10. SHDI distribution maps for study area from 2016-2020. 


\section{Discussion \\ 4.1. Spatial Correlation between UGS and Population Density}

The UGI represents proportional green cover near each pixel within the whole study area. Taking spatial variations in population density into account in the descriptive statistics of the UGI value allows the indicators to represent the perspectives of citizens, which may be more meaningful for government administration.

We connected the UGI map with the population density map for 2020 to identify the population sizes according to the different levels of surrounding greenness, especially in conditions of poor vegetation cover (Figure 11). Hot spot analysis was used to identify the spatial clustering that had high values (hot spots) and low values (cold spots) [63].

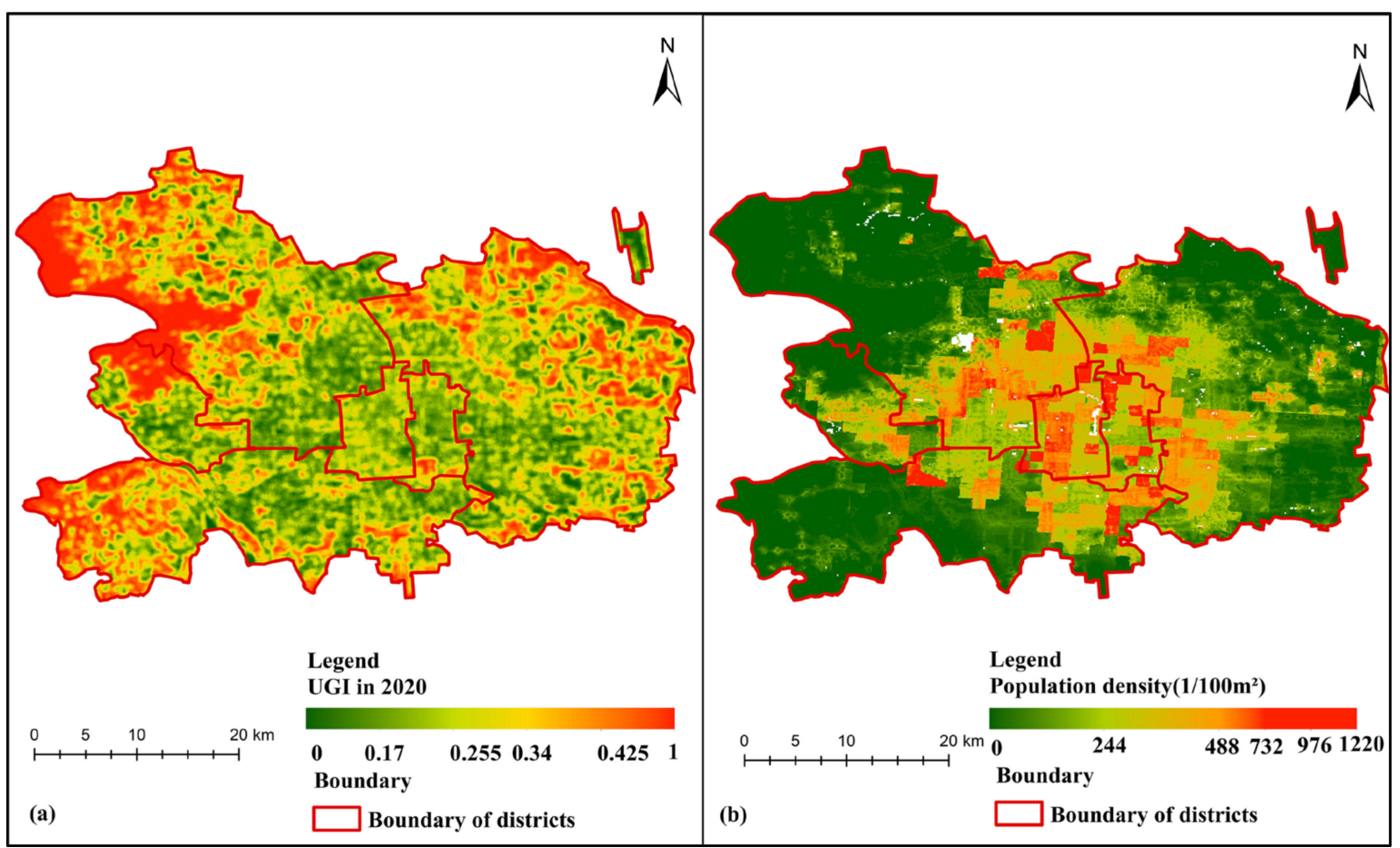

Figure 11. (a) Spatial distribution of UGI mapand (b) population density map for 2020.

Based on the hot spot analysis with rendering (Getis-Ord $\mathrm{Gi}^{*}$ ) offered by ArcGIS, we calculated the Getis-Ord $\mathrm{Gi}^{*}$ statistics for the input layer, the 2020 UGI value, and the population density grid for this study. We identified statistically significant spatial clusters with hot spots (high UGI value and high population density) and cold spots (low UGI value and low population density). Then, we identified five UGI cold spots in the area of high population density. Three of the five cold spots were located in the east and southeast of Haidian District (Figure 12). The hot spots of the population and UGI were spatially inconsistent.

Therefore, it is necessary to understand the vegetation cover around a density population pixel. To distinguish the difference in a population pixel's UGI value, according to the ratio of the UGI value of each population pixel to the average UGI value of the entire population, the average UGI value of the entire population pixel was determined to be 0.34 in 2020. The UGI values of the population pixel were divided into five grades: lowest $(0.00-0.17)$, low $(0.17-0.25)$, medium $(0.25-0.34)$, high $(0.34-0.42)$, and highest $(0.42-1.00)$ (Table 7). Figure 13 shows the proportion density of residents with different UGI levels in the six districts. 


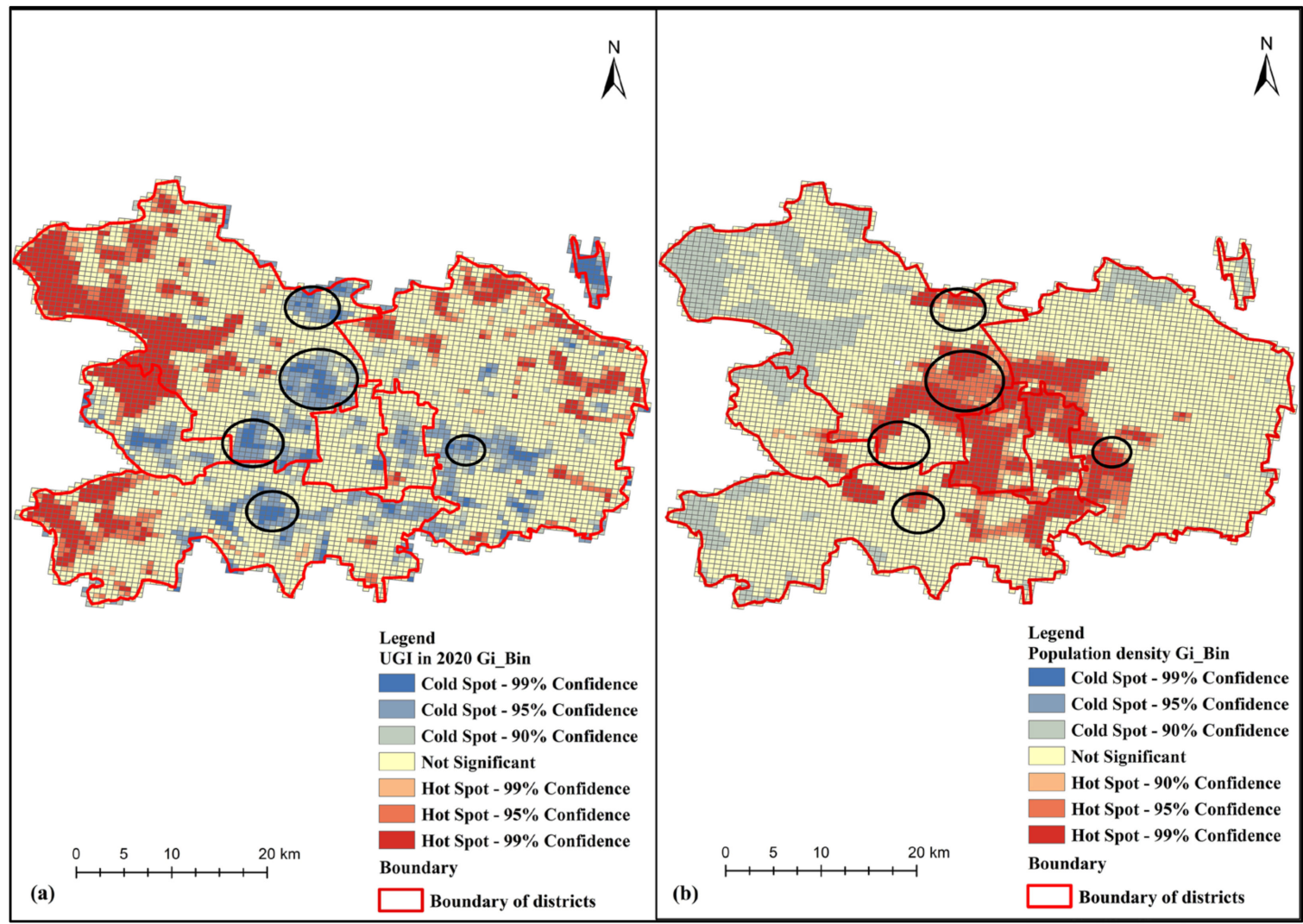

Figure 12. (a) Spatial distribution of the hot and cold spots of UGI and (b) population density in the center of Beijing in 2020.

Table 7. Population statistics of different districts with different UGI values.

\begin{tabular}{cccccc}
\hline UGI & Lowest & Low & Medium & High & Highest \\
\hline Chaoyang & 369,478 & 599,888 & 664,165 & 524,885 & $1,275,174$ \\
Dongcheng & 49,036 & 186,980 & 264,324 & 152,743 & 195,402 \\
Fengtai & 265,572 & 383,028 & 418,559 & 306,491 & 706,531 \\
Haidian & 451,659 & 675,769 & 600,579 & 414,273 & 929,945 \\
Shijingshan & 79,478 & 125,627 & 120,443 & 97,853 & 181,602 \\
Xicheng & 30,399 & 132,756 & 362,650 & 346,407 & 324,878 \\
\hline
\end{tabular}

As can be seen from Figure 13, the proportion of residents of Chaoyang and Xicheng in the high and highest levels was more than $50 \%$ (52\% and $56 \%$, respectively), while residents in the lowest or low levels accounted for $28 \%$ and $13 \%$, respectively. It can be seen that most residents were situated in areas with good vegetation coverage in these two districts. Up to $57 \%$ and $54 \%$ of residents were situated in areas with medium and below vegetation coverage in Haidian and Shijingshan, while the area ratio for these two levels was only $29 \%$ and $34 \%$, respectively. Therefore, considering the factor of population density, the layout of green space in these two districts should be optimized. 


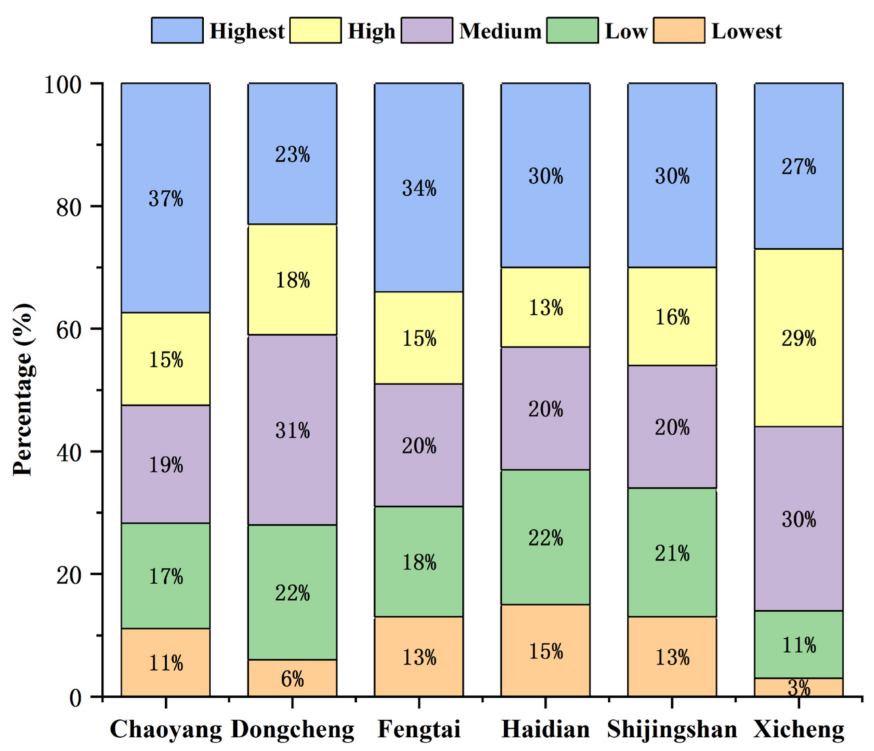

Figure 13. The proportion of the population with different UGI levels in six districts.

\subsection{Distinction between Private and Public Green Spaces}

The United Nations Sustainable Development Goal (SDG) indicator 11.7.1 requires the following: "by 2030, provide universal access to safe, inclusive and accessible, green and public spaces, particularly for women and children, older persons and persons with disabilities". It calls on city managers to pay attention to the amount of land designated for public spaces. For cities in the developing world, some of the proposed SDG indicators are currently on hold due to a lack of data. It is critical for these cities to monitor public green spaces using free data sources. Without distinguishing between public and private green spaces, we assessed the quality of UGS as perceived by residents on a 4 min walk (300 m) (Section 3.3). We assessed the accessibility of urban public green spaces in order to determine whether they comply with the SDG11.7.1. Firstly, we used crowdsourced data from OpenStreetMap (OSM) and adapted the methodology proposed by Ludwig et al. [64] to capture public areas. Then, the layer of public areas was intersected together with the UGS map to identify green areas that were open to the public. Therefore, the total public green spaces were estimated (Figure 14).

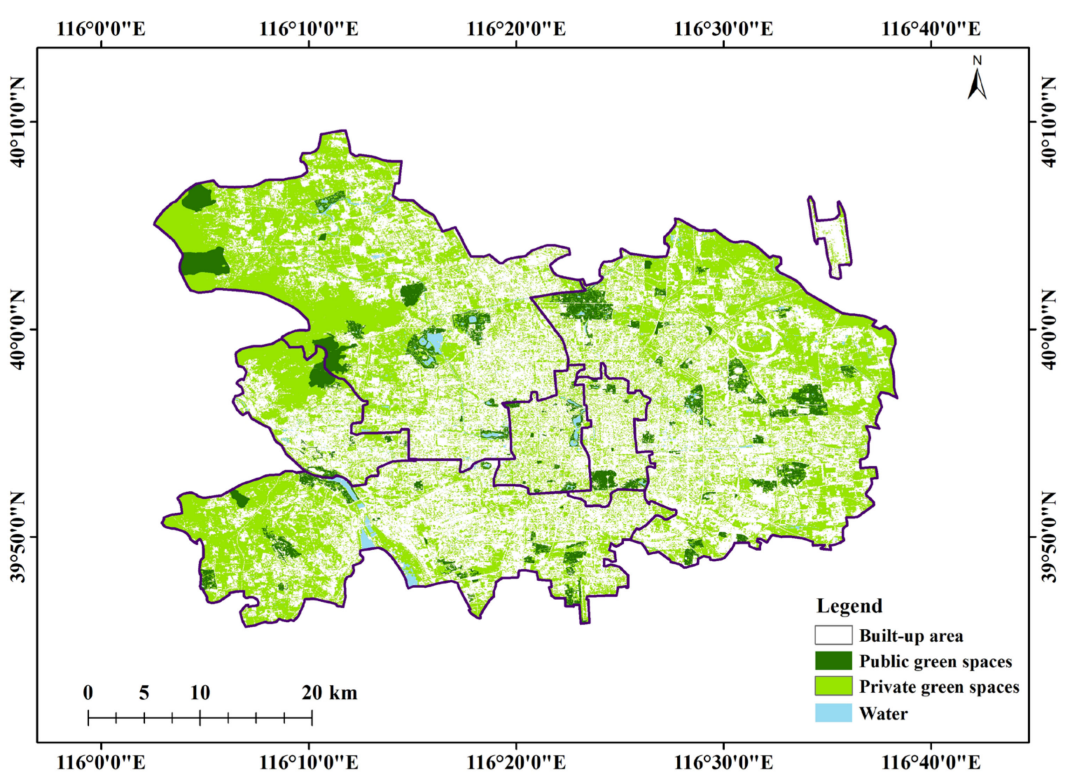

Figure 14. Distribution of public and private green spaces in 2020. 
We used the improved gravity model [65] to measure the accessibility to public green spaces. The improved gravity model is shown:

$$
\begin{aligned}
A_{i} & =\sum_{j} \frac{S_{j}}{V_{j} d_{i j}^{\beta}} \\
V_{j} & =\sum_{k} \frac{P_{k}}{d_{k j}^{\beta}}
\end{aligned}
$$

where $A_{i}$ is the spatial accessibility from population point $i ; S_{j}$ is the service capacity at provider location $j ; d$ is the travel impedance, e.g., distance or travel time between points $i$ and $j ; \beta$ is a gravity decay coefficient; $V_{j}$ is the population demand adjustment factor; and $P_{k}$ is the population size at point $k$.

Another commonly used method for evaluating accessibility is the two-step floating catchment area method (2SFCA). The two models consider the scale of both the supply and demand of facilities. However, the 2SFCA cannot take into account the decreasing service capability of facilities over a distance [66]. The urban public green spaces are classified based on their sizes and are assigned with different service radii accordingly: a municipal park, with an area exceeding $10 \mathrm{hm}^{2}$, serves the entire city and the adjacent area at a radius of $3000 \mathrm{~m}$; a district park, with an area of $5-10 \mathrm{hm}^{2}$, has a service radius of $1500 \mathrm{~m}$; and a residential area park, with an area of $0.5-5 \mathrm{hm}^{2}$, has a service radius of $750 \mathrm{~m}$. The commonly used improved gravity model does not set a limit on the effective search radius of a facility. We improved this model by adding the influence coefficient $M_{i j}$ of the park grade scale. The formula is as follows:

$$
\begin{aligned}
A_{i} & =\sum_{j} \frac{M_{i j} S_{j}}{V_{j} d_{i j}^{\beta}} \\
V_{j} & =\sum_{k} \frac{M_{k j} P_{k}}{d_{k j}^{\beta}}
\end{aligned}
$$

where $A_{i}$ is the spatial accessibility from population point $i, S_{j}$ is the area size of the park green space $j, d_{i j}$ is the travel distance between points $i$ and $\mathbf{j}$, and $\beta$ is a gravity decay coefficient. In our study, $\beta$ is equal to 1 and $M_{i j}$ is equal to 1 when $d_{i j}$ is less than the service radius of the park $j$; otherwise, $M_{i j}$ is equal to $0 . V_{j}$ is the population demand adjustment factor, $P_{k}$ is the population size at point $k$, and $M_{k j}$ is equal to 1 when $d_{k j}$ is less than the service radius of the park $j$; otherwise, $M_{i j}$ is equal to 0 .

Then, we mapped the accessibility of central Beijing according to the improved gravity model for 2020 (Figure 15). The population accessibility to public green spaces considering both the service capacity of the park and the demand of the population shows important differences in spatial distribution. In general, the accessibility of Dongcheng and Xicheng was lower than that of the other four districts, and the lowest accessibility was located in the southeast of Haidian District and the north of Fengtai District. In order to achieve green equity, it is necessary to increase some public green spaces in areas with poor accessibility, such as street corner parks and community parks. 


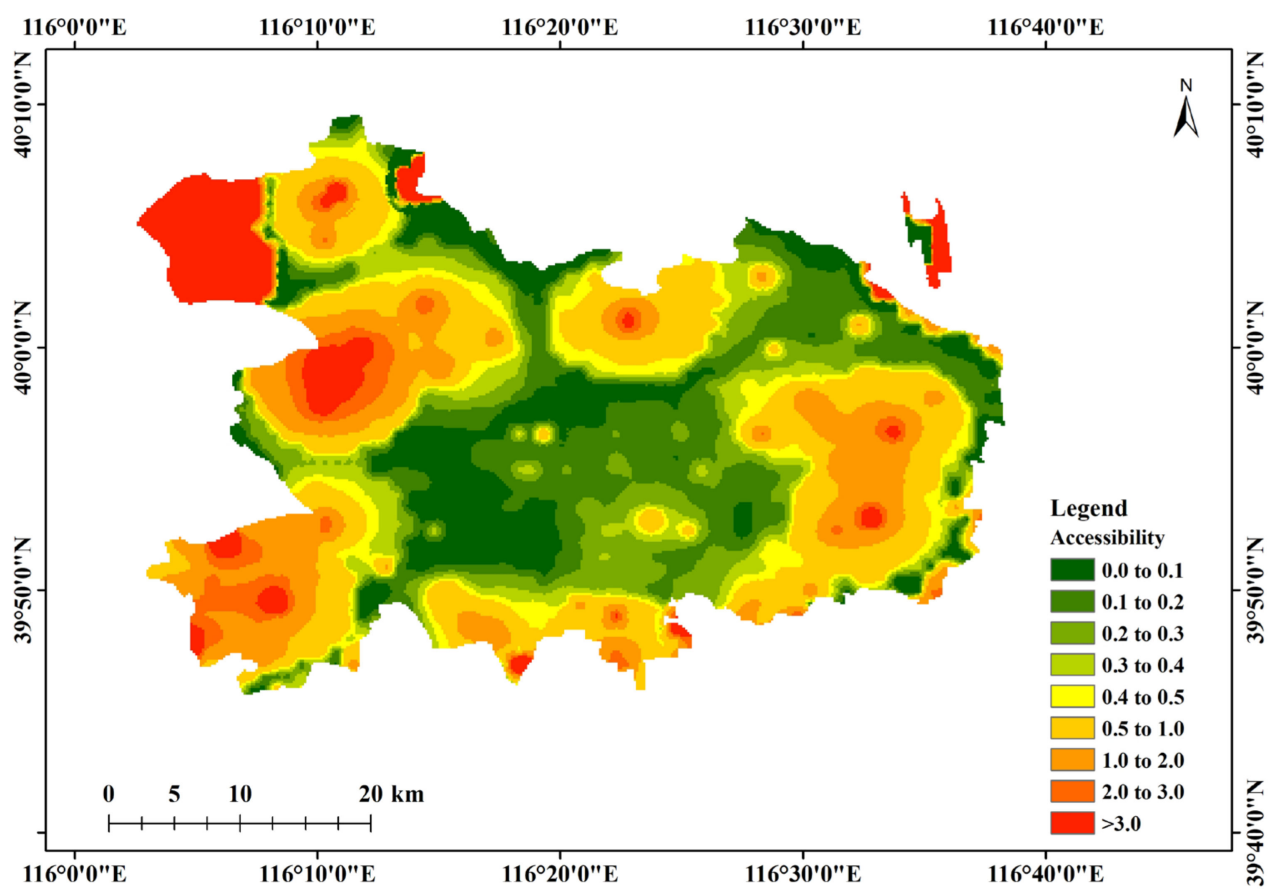

Figure 15. Public green spaces accessibility map for the center of Beijing in 2020.

\section{Conclusions}

In this study, annual UGS maps with a $10 \mathrm{~m}$ spatial resolution were produced for the center of Beijing. To this end, seasonal Sentinel-2 data from 21 June to 21 September, collected from 2016 to 2020, were employed. The GEE platform was used to rapidly and automatically update the UGS map for any target year. Multiple features extracted from Sentinel-2 images were input into the RF classifier for the differentiation of vegetation types. The obtained average OA of $96.47 \%$ demonstrated the high potential of the proposed GEE-based automatic mapping method for the production of up-to-date UGS maps, enabling users, such as relevant governmental administrative departments, to investigate the dynamics of UGS over longer periods.

We evaluated the distribution of UGS in the center of Beijing from 2016 to 2020. Six landscape pattern metrics were adopted to assess the quality of UGSdistribution in order to provide a thorough understanding of the dynamics of UGS distribution. We found that most of the districts in the center of Beijing improved their vegetation cover condition and diversity in a fluctuating way. However, the distribution of UGS and population was not consistent; for example, in Haidian District in 2020, the area where the UGI was value below 0.34 accounted for $29 \%$, but its population accounted for $56 \%$. We revealed that the diversity of green spaces tended to be more balanced. Furthermore, the public green spaces accessibility map of central Beijing in 2020 showed that the lowest accessibility was located in the southeast of Haidian District and the north of Fengtai District.

The proposed workflow combines multiple approaches, time series Sentinel-2 images, and the GEE platform is proved to be effective in the dynamic mapping and evaluation of UGS. The workflow has great potential to be applied to the detection of vegetation cover dynamics for other cities in a cost-effective manner. This would be particularly useful in the case of cities in developing countries that exhibit high urban dynamism spatially and temporally. However, to track the long-term dynamics of UGSs, it is necessary to integrate other satellite data, such as the Landsat imagery, because the multispectral images from Sentinel-2A have been available since 2015 when the Sentinel-2A satellite was initially launched. In addition, to monitor UGS for cloud-rich areas, microwave images from Sentinel-1 can be combined with optical images. Further, our work shows that the proposed workflow can be further 
combined with other free geospatial data and accessibility models to support evidence-based decision making for sustainable urban development.

Author Contributions: Conceptualization, Qiang Chen and Changfeng Jing; data curation, Beilei Cao; formal analysis, Qiang Chen and Changfeng Jing; funding acquisition, Qiang Chen; investigation, Cuiping Zhong; methodology, Cuiping Zhong; project administration, Qiang Chen; resources, Yuanyuan Li; software, Cuiping Zhong; supervision, Changfeng Jing; validation, Qiang Chen; visualization, Yuanyuan Li and Qianhao Cheng; writing — original draft, Cuiping Zhong; writing-review and editing, Qiang Chen and Yuanyuan Li. All authors have read and agreed to the published version of the manuscript.

Funding: This research was funded by the National Natural Science Foundation of China (41801235), General Scientific Research Project of Beijing Educational Committee (KM20190016006), and The Pyramid Talent Training Project of Beijing University of Civil Engineering and Architecture (JDJQ20200306).

Institutional Review Board Statement: Not applicable.

Informed Consent Statement: Not applicable.

Data Availability Statement: The data that support the findings of this study are available from the author upon reasonable request.

Acknowledgments: The authors would like to thank the editors and anonymous reviewers for their valuable time and efforts in reviewing this manuscript.

Conflicts of Interest: The authors declare no conflict of interest.

\section{Appendix A}

Table A1. Spectral features list.

\begin{tabular}{ccc}
\hline Name & Abbreviation & Description \\
\hline Spectral value & B2 & Blue band \\
& B3 & Green band \\
Bed band & Red Edge band 1 \\
& B5 & Red Edge band 2 \\
B6 & Red Edge band 3 \\
B7 & NIR band \\
Hue & Hue & HSI color space Hue \\
Intensity & Int & Brightness of HSI color space \\
Brightness & Rat1 & Blue band contribution (ratio of Blue band spectral values to \\
Contribution rate & the sum of all band spectral values) \\
& Rat2 & Red band contribution (ratio of Red band spectral values to \\
the sum of all band spectral values)
\end{tabular}


Table A2. Vegetation indices list.

\begin{tabular}{cc}
\hline Vegetation Indices & Formula \\
\hline $\begin{array}{c}\text { Normalized Difference Vegetation Index } \\
\text { (NDVI) }\end{array}$ & NDVI $=\frac{N I R-R}{N I R+R}$ \\
Greenness index (GI) & $\mathrm{GI}=\frac{G}{R}$ \\
Ratio vegetation index (RVI) & $\mathrm{RVI}=\frac{N I R}{R}$ \\
Difference vegetation index (DVI) & $\mathrm{DVI}=\mathrm{NIR}-\mathrm{R}$ \\
Soil-adjusted vegetation index (SAVI) & SAVI $=\frac{(N I R-R)(L+1)}{N I R+R+L}$ \\
$\begin{array}{l}\text { Note: } N I R \text { is the reflectance of the near-infrared band (band } 8, \text { central wavelength: } 842 \mathrm{~nm}), R \text { is the reflectance } \\
\text { of the Red band (band } 4 \text {, central wavelength: } 665 \mathrm{~nm}), G \text { is the reflectance of the Green band (band 3, central } \\
\text { wavelength: } 560 \mathrm{~nm}), L \text { is the soil-adjusted parameter, and } L=0.5 \text { in our study area. }\end{array}$
\end{tabular}

Table A3. GLCM indices list.

\begin{tabular}{|c|c|c|}
\hline Number & Features & Formula \\
\hline 1 & Angular Second Moment (ASM) & $P_{1}=\sum_{i} \sum_{i}(f(i, j))^{2}$ \\
\hline 2 & CONTRAST (Contrast) & $P_{2}=\sum_{n=0}^{N_{g-1}} n^{2}\left(\begin{array}{c}\sum_{i=1}^{N_{g}} \sum_{j=1}^{N_{g}} f(i, j) \\
|i-j|=n\end{array}\right)$ \\
\hline 3 & Correlation (CORR) & $P_{3}=\frac{\sum_{i} \sum_{j}(i j) f(i j)-\mu_{x} \mu_{y}}{\sigma_{x} \sigma_{y}}$ \\
\hline 4 & Variance (VAR) & $P_{4}=\sum_{i} \sum_{j}(i-u)^{2} f(i, j)$ \\
\hline 5 & Difference Moment (IDM) & $P_{5}=\sum_{i} \sum_{j} \frac{1}{1+(i-j)^{2}} f(i, j)$ \\
\hline 6 & Sum Average (SAVG) & $P_{6}=\sum_{i=2}^{2 N_{g}} i f_{x+y}(i)$ \\
\hline 7 & Sum Variance (SVAR) & $P_{7}=\sum_{i=2}^{2 N_{g}}\left(i-P_{8}\right)^{2} f_{x+y}(i)$ \\
\hline 8 & Sum Entropy (SENT) & $P_{8}=-\sum_{i=2}^{2 N_{g}} \log \left(f_{x+y}(i)\right) f_{x+y}(i)$ \\
\hline 9 & Entropy (ENT) & $P_{9}=-\sum_{i} \sum_{j} f(i, j) \log (f(i, j))$ \\
\hline 10 & DVAR, Difference variance (DVAR) & $P_{10}=$ variance of $f_{x-y}$ \\
\hline 11 & $\begin{array}{l}\text { Difference entropy } \\
\text { (DENT) }\end{array}$ & $P_{11}=-\sum_{i}^{N_{g}-1} f_{x-y}(i) \log \left\{f_{x-y}(i)\right\}$ \\
\hline 12 & $\begin{array}{l}\text { Information Measure of Corr. } 1 \\
\text { (IMCORR1) }\end{array}$ & $P_{12}=\frac{H X Y-H X Y 1}{\max \{H X, H Y\}}$ \\
\hline 13 & $\begin{array}{l}\text { Information Measure of Corr. } 2 \\
\text { (IMORR2) }\end{array}$ & $P_{13}=\left(1-\exp [-2.0(H X Y 2-H X Y)]^{\frac{1}{2}}\right.$ \\
\hline 14 & Maximal Correlation Coefficient & $\begin{array}{c}P_{14}=(\text { Second largest eigenvalue of } Q)^{\frac{1}{2}} \\
Q_{(i, j)}=\sum_{k} \frac{f(i, k) f(j, k)}{f_{x}(i) f_{y}(i)}\end{array}$ \\
\hline 15 & $\begin{array}{l}\text { Dissimilarity } \\
\quad \text { (DISS) }\end{array}$ & $\mathrm{L}(\delta, \mathrm{T})=\sum_{i=0}^{L-1} \sum_{j=0}^{L-1} \frac{1}{1+(i-j)^{2}} s(i, j, \delta, T)$ \\
\hline
\end{tabular}


Table A3. Cont.

\begin{tabular}{|c|c|c|}
\hline Number & Features & Formula \\
\hline 16 & $\begin{array}{c}\text { Inertia } \\
\text { (INERTIA) }\end{array}$ & $\mathrm{I}(\delta, \mathrm{T})=\sum_{i=0}^{L-1} \sum_{J=0}^{L-1}(i-j)^{2} s(i, j, \delta, T)$ \\
\hline 17 & Cluster Shade (SHADE) & $\begin{array}{c}\mathrm{A}(\delta, \mathrm{T})= \\
\sum_{i=0}^{L-1} \sum_{j=0}^{L-1}\left(i+j-\mu_{i}-\mu_{j}\right)^{3} s(i, j, \delta, T)\end{array}$ \\
\hline 18 & Cluster prominence (PROM) & $\begin{array}{c}\mathrm{B}(\delta, \mathrm{T})= \\
\sum_{i=0}^{L-1} \sum_{j=0}^{L-1}\left(i+j-\mu_{i}-\mu_{j}\right)^{4} s(i, j, \delta, T)\end{array}$ \\
\hline
\end{tabular}

Note: $f(i j)(i, j)$ th entry in a normalized gray-tone spatial-dependence matrix. $f_{x}(i) i$ th entry in the marginal probability matrix obtained by summing the rows of $f(i j),=\sum_{j=1}^{N_{g}} f(i, j) . N_{g}$ Number of distinct gray levels in the quantized image. $\mu_{x}, \mu_{y}, \sigma_{x}$ and $\sigma_{y}$ are the means and standard deviations of $f_{x}$ and $f_{y} \cdot f_{y}(j)=\sum_{i=1}^{N_{g}} f(i, j) \cdot f_{x+y}(k)=\sum_{i=1}^{N_{g}} \sum_{j=1}^{N_{g}} f(i, j)$, $i+j=k, k=2,3, \cdots, 2 N_{g} \cdot f_{x-y}(k)=\sum_{i=1}^{N_{g}} \sum_{j=1}^{N_{g}} f(i, j),|i-j|=k, k=0,1, \cdots, N_{g}-1$.

For more details, see [37,67].

\section{References}

1. Meng, Q.; Zhang, L.; Sun, Z.; Meng, F.; Wang, L.; Sun, Y. Characterizing spatial and temporal trends of surface urban heat island effect in an urban main built-up area: A 12-year case study in Beijing, China. Remote Sens. Environ. 2018, 204, 826-837. [CrossRef]

2. Lo, A.Y.; Jim, C.Y. Citizen attitude and expectation towards greenspace provision in compact urban milieu. Land Use Policy 2012, 29, 577-586. [CrossRef]

3. Sathyakumar, V.; Ramsankaran, R.; Bardhan, R. Geospatial approach for assessing spatiotemporal dynamics of urban green space distribution among neighbourhoods: A demonstration in Mumbai. Urban For. Urban Green. 2020, 48, 126585. [CrossRef]

4. $\quad$ Liu, Q.; Tian, Y.; Yin, K.; Zhang, F.; Huang, H.; Chen, F. Landscape Pattern Theoretical Optimization of Urban Green Space Based on Ecosystem Service Supply and Demand. ISPRS Int. J. Geo-Inf. 2021, 10, 263. [CrossRef]

5. Schebella, M.; Weber, D.; Schultz, L.; Weinstein, P. The Wellbeing Benefits Associated with Perceived and Measured Biodiversity in Australian Urban Green Spaces. Sustainability 2019, 11, 802. [CrossRef]

6. Chun, B.; Guldmann, J.-M. Impact of greening on the urban heat island: Seasonal variations and mitigation strategies. Comput. Environ. Urban Syst. 2018, 71, 165-176. [CrossRef]

7. Shin, J.C.; Kwan, M.P.; Grigsby-Toussaint, D.S. Do Spatial Boundaries Matter for Exploring the Impact of Community Green Spaces on Health? Int. J. Environ. Res Public Health 2020, 17, 7529. [CrossRef]

8. Wiese, D.; Stroup, A.M.; Maiti, A.; Harris, G.; Lynch, S.M.; Vucetic, S.; Gutierrez-Velez, V.H.; Henry, K.A. Measuring Neighborhood Landscapes: Associations between a Neighborhood's Landscape Characteristics and Colon Cancer Survival. Int. J. Environ. Res. Public Health 2021, 18, 4728. [CrossRef]

9. Xie, Y.; Xiang, H.; Di, N.; Mao, Z.; Hou, J.; Liu, X.; Huo, W.; Yang, B.; Dong, G.; Wang, C. Association between residential greenness and sleep quality in Chinese rural population. Environ. Int. 2020, 145, 106100. [CrossRef]

10. De Sousa Silva, C.; Viegas, I.; Panagopoulos, T.; Bell, S. Environmental justice in accessibility to green infrastructure in two European cities. Land 2018, 7, 134. [CrossRef]

11. Reid, C.E.; Kubzansky, L.D.; Li, J.; Shmool, J.L.; Clougherty, J.E. It's not easy assessing greenness: A comparison of NDVI datasets and neighborhood types and their associations with self-rated health in New York City. Health Place 2018, 54, 92-101. [CrossRef]

12. Fan, P.; Xu, L.; Yue, W.; Chen, J. Accessibility of public urban green space in an urban periphery: The case of Shanghai. Landsc. Urban Plan. 2017, 165, 177-192. [CrossRef]

13. Liu, S.; Su, H.; Cao, G.; Wang, S.; Guan, Q. Learning from data: A post classification method for annual land cover analysis in urban areas. ISPRS J. Photogramm. Remote Sens. 2019, 154, 202-215. [CrossRef]

14. Ji, J.S.; Zhu, A.; Bai, C.; James, P.; Yan, L.; Zeng, Y.; Wu, C.-D. Residential greenness and mortality in oldest-old women and men in China: A prospective cohort study. Lancet 2018, 392, S65. [CrossRef]

15. Rugel, E.J.; Carpiano, R.M.; Henderson, S.B.; Brauer, M. Exposure to natural space, sense of community belonging, and adverse mental health outcomes across an urban region. Environ. Res. 2019, 171, 365-377. [CrossRef]

16. Yang, L.; Ho, J.Y.; Wong, F.K.; Chang, K.K.; Chan, K.L.; Wong, M.S.; Ho, H.C.; Yuen, J.W.; Huang, J.; Siu, J.Y. Neighbourhood green space, perceived stress and sleep quality in an urban population. Urban For. Urban Green. 2020, 54, 126763. [CrossRef]

17. Ghorbanian, A.; Kakooei, M.; Amani, M.; Mahdavi, S.; Mohammadzadeh, A.; Hasanlou, M. Improved land cover map of Iran using Sentinel imagery within Google Earth Engine and a novel automatic workflow for land cover classification using migrated training samples. ISPRS J. Photogramm. Remote Sens. 2020, 167, 276-288. [CrossRef] 
18. Mahdianpari, M.; Salehi, B.; Mohammadimanesh, F.; Homayouni, S.; Gill, E. The First Wetland Inventory Map of Newfoundland at a Spatial Resolution of $10 \mathrm{~m}$ Using Sentinel-1 and Sentinel-2 Data on the Google Earth Engine Cloud Computing Platform. Remote Sens. 2018, 11, 43. [CrossRef]

19. Huang, H.; Wang, J.; Liu, C.; Liang, L.; Li, C.; Gong, P. The migration of training samples towards dynamic global land cover mapping. ISPRS J. Photogramm. Remote Sens. 2020, 161, 27-36. [CrossRef]

20. Li, Q.; Qiu, C.; Ma, L.; Schmitt, M.; Zhu, X.X. Mapping the land cover of Africa at $10 \mathrm{~m}$ resolution from multi-source remote sensing data with Google Earth Engine. Remote Sens. 2020, 12, 602. [CrossRef]

21. Wang, Y.; Li, Z.; Zeng, C.; Xia, G.-S.; Shen, H. An urban water extraction method combining deep learning and google earth engine. IEEE J. Sel. Top. Appl. Earth Obs. Remote Sens. 2020, 13, 769-782. [CrossRef]

22. Bullock, E.L.; Woodcock, C.E.; Olofsson, P. Monitoring tropical forest degradation using spectral unmixing and Landsat time series analysis. Remote Sens. Environ. 2020, 238, 110968. [CrossRef]

23. Xiong, J.; Thenkabail, P.S.; Gumma, M.K.; Teluguntla, P.; Poehnelt, J.; Congalton, R.G.; Yadav, K.; Thau, D. Automated cropland mapping of continental Africa using Google Earth Engine cloud computing. ISPRS J. Photogramm. Remote Sens. 2017, 126, 225-244. [CrossRef]

24. Praticò, S.; Solano, F.; Di Fazio, S.; Modica, G. Machine Learning Classification of Mediterranean Forest Habitats in Google Earth Engine Based on Seasonal Sentinel-2 Time-Series and Input Image Composition Optimisation. Remote Sens. 2021, 13, 586. [CrossRef]

25. Coltin, B.; McMichael, S.; Smith, T.; Fong, T. Automatic boosted flood mapping from satellite data. Int. J. Remote Sens. 2016, 37, 993-1015. [CrossRef] [PubMed]

26. Mahdianpari, M.; Brisco, B.; Granger, J.E.; Mohammadimanesh, F.; Salehi, B.; Banks, S.; Homayouni, S.; Bourgeau-Chavez, L.; Weng, Q. The second generation Canadian wetland inventory map at 10 meters resolution using Google Earth Engine. Can. J. Remote Sens. 2020, 46, 360-375. [CrossRef]

27. Huang, C.; Yang, J.; Clinton, N.; Yu, L.; Huang, H.; Dronova, I.; Jin, J. Mapping the maximum extents of urban green spaces in 1039 cities using dense satellite images. Environ. Res. Lett. 2021, 16, 064072. [CrossRef]

28. Huang, C.; Yang, J.; Jiang, P. Assessing impacts of urban form on landscape structure of urban green spaces in China using Landsat images based on Google Earth Engine. Remote Sens. 2018, 10, 1569. [CrossRef]

29. Duan, Q.; Tan, M.; Guo, Y.; Wang, X.; Xin, L. Understanding the spatial distribution of urban forests in China using Sentinel-2 images with Google Earth Engine. Forests 2019, 10, 729. [CrossRef]

30. Gilani, H.; Naz, H.I.; Arshad, M.; Nazim, K.; Akram, U.; Abrar, A.; Asif, M. Evaluating mangrove conservation and sustainability through spatiotemporal (1990-2020) mangrove cover change analysis in Pakistan. Estuar. Coast. Shelf Sci. 2021, 249. [CrossRef]

31. Stessens, P.; Khan, A.Z.; Huysmans, M.; Canters, F. Analysing urban green space accessibility and quality: A GIS-based model as spatial decision support for urban ecosystem services in Brussels. Ecosyst. Serv. 2017, 28, 328-340. [CrossRef]

32. Honold, J.; Lakes, T.; Beyer, R.; van der Meer, E. Restoration in urban spaces: Nature views from home, greenways, and public parks. Environ. Behav. 2016, 48, 796-825. [CrossRef]

33. Atasoy, M. Monitoring the urban green spaces and landscape fragmentation using remote sensing: A case study in Osmaniye, Turkey. Environ. Monit. Assess. 2018, 190, 713. [CrossRef]

34. Tamiminia, H.; Salehi, B.; Mahdianpari, M.; Quackenbush, L.; Adeli, S.; Brisco, B. Google Earth Engine for geo-big data applications: A meta-analysis and systematic review. ISPRS J. Photogramm. Remote Sens. 2020, 164, 152-170. [CrossRef]

35. Myint, S.W.; Gober, P.; Brazel, A.; Grossman-Clarke, S.; Weng, Q. Per-pixel vs. object-based classification of urban land cover extraction using high spatial resolution imagery. Remote Sens. Environ. 2011, 115, 1145-1161. [CrossRef]

36. Ouma, Y.O.; Ngigi, T.; Tateishi, R. On the optimization and selection of wavelet texture for feature extraction from high-resolution satellite imagery with application towards urban-tree delineation. Int. J. Remote Sens. 2006, 27, 73-104. [CrossRef]

37. Haralick, R.M.; Shanmugam, K.; Dinstein, I.H. Textural features for image classification. IEEE Trans. Syst. Man Cybern. 1973, 610-621. [CrossRef]

38. Hidayat, S.; Matsuoka, M.; Baja, S.; Rampisela, D.A. Object-based image analysis for sago palm classification: The most important features from high-resolution satellite imagery. Remote Sens. 2018, 10, 1319. [CrossRef]

39. Otsu, N. A threshold selection method from gray-level histograms. IEEE Trans. Syst. Man Cybern. 1979, 9, 62-66. [CrossRef]

40. Jia, M.; Wang, Z.; Mao, D.; Ren, C.; Wang, C.; Wang, Y. Rapid, robust, and automated mapping of tidal flats in China using time series Sentinel-2 images and Google Earth Engine. Remote Sens. Environ. 2021, 255, 112285. [CrossRef]

41. Donchyts, G.; Baart, F.; Winsemius, H.; Gorelick, N.; Kwadijk, J.; Van De Giesen, N. Earth's surface water change over the past 30 years. Nat. Clim. Chang. 2016, 6, 810-813. [CrossRef]

42. Maxwell, A.E.; Warner, T.A.; Fang, F. Implementation of machine-learning classification in remote sensing: An applied review. Int. J. Remote Sens. 2018, 39, 2784-2817. [CrossRef]

43. Le, Q.I.; Yue, C.R. Remote Sensing Image Classification Based on CART Decision Tree Method. For. Inventory Plan. 2011, 36, 62-66.

44. Ustuner, M.; Sanli, F.B.; Dixon, B. Application of Support Vector Machines for Landuse Classification Using High-Resolution RapidEye Images: A Sensitivity Analysis. Eur. J. Remote Sens. 2017, 48, 403-422. [CrossRef]

45. Kranjčić, N.; Medak, D.; Župan, R.; Rezo, M. Support vector machine accuracy assessment for extracting green urban areas in towns. Remote Sens. 2019, 11, 655. [CrossRef] 
46. Phan, T.N.; Kuch, V.; Lehnert, L.W. Land Cover Classification using Google Earth Engine and Random Forest Classifier-The Role of Image Composition. Remote Sens. 2020, 12, 2411. [CrossRef]

47. Sarah, B.; Koreen, M.; Jon, P.; Murray, R.; Wang, H.; Jason, D. Assessing the Potential to Operationalize Shoreline Sensitivity Mapping: Classifying Multiple Wide Fine Quadrature Polarized RADARSAT-2 and Landsat 5 Scenes with a Single Random Forest Model. Remote Sens. 2015, 7, 13528-13563.

48. Du, L.; Feng, Y.; Lu, W.; Kong, L.; Yang, Z. Evolutionary game analysis of stakeholders' decision-making behaviours in construction and demolition waste management. Environ. Impact Assess. Rev. 2020, 84, 106408. [CrossRef]

49. Mohammadimanesh, F.; Salehi, B.; Mahdianpari, M.; Brisco, B.; Gill, E. Full and simulated compact polarimetry sar responses to canadian wetlands: Separability analysis and classification. Remote Sens. 2019, 11, 516. [CrossRef]

50. Gu, H.; Li, H.; Yan, L.; Liu, Z.; Blaschke, T.; Soergel, U. An object-based semantic classification method for high resolution remote sensing imagery using ontology. Remote Sens. 2017, 9, 329. [CrossRef]

51. Congalton, R.G. A review of assessing the accuracy of classifications of remotely sensed data. Remote Sens. Environ. 1991, 37, 35-46. [CrossRef]

52. Shaharum, N.S.N.; Shafri, H.Z.M.; Ghani, W.A.W.A.K.; Samsatli, S.; Prince, H.M.; Yusuf, B.; Hamud, A.M. Mapping the spatial distribution and changes of oil palm land cover using an open source cloud-based mapping platform. Int. J. Remote Sens. 2019, 40, 7459-7476. [CrossRef]

53. Li, C.; Wang, J.; Wang, L.; Hu, L.; Gong, P. Comparison of classification algorithms and training sample sizes in urban land classification with Landsat thematic mapper imagery. Remote Sens. 2014, 6, 964-983. [CrossRef]

54. Amani, M.; Brisco, B.; Afshar, M.; Mirmazloumi, S.M.; Mahdavi, S.; Mirzadeh, S.M.J.; Huang, W.; Granger, J. A generalized supervised classification scheme to produce provincial wetland inventory maps: An application of Google Earth Engine for big geo data processing. Big Earth Data 2019, 3, 378-394. [CrossRef]

55. Zhang, H.K.; Roy, D.P. Using the $500 \mathrm{~m}$ MODIS land cover product to derive a consistent continental scale $30 \mathrm{~m}$ Landsat land cover classification. Remote Sens. Environ. 2017, 197, 15-34. [CrossRef]

56. Radoux, J.; Lamarche, C.; Van Bogaert, E.; Bontemps, S.; Brockmann, C.; Defourny, P. Automated training sample extraction for global land cover mapping. Remote Sens. 2014, 6, 3965-3987. [CrossRef]

57. Ji, L.; Geng, X.; Sun, K.; Zhao, Y.; Gong, P. Target detection method for water mapping using Landsat 8 OLI/TIRS imagery. Water 2015, 7, 794-817. [CrossRef]

58. Carvalho Júnior, O.A.; Guimarães, R.F.; Gillespie, A.R.; Silva, N.C.; Gomes, R.A. A new approach to change vector analysis using distance and similarity measures. Remote Sens. 2011, 3, 2473-2493. [CrossRef]

59. Haaland, C.; van Den Bosch, C.K. Challenges and strategies for urban green-space planning in cities undergoing densification: A review. Urban For. Urban Green. 2015, 14, 760-771. [CrossRef]

60. De la Barrera, F.; Reyes-Paecke, S.; Banzhaf, E. Indicators for green spaces in contrasting urban settings. Ecol. Indic. 2016, 62, 212-219. [CrossRef]

61. Hong, K.Y.; Tsin, P.K.; van den Bosch, M.; Brauer, M.; Henderson, S.B. Urban greenness extracted from pedestrian video and its relationship with surrounding air temperatures. Urban For. Urban Green. 2019, 38, 280-285. [CrossRef]

62. You, H. Characterizing the inequalities in urban public green space provision in Shenzhen, China. Habitat Int. 2016, 56, 176-180. [CrossRef]

63. Kondo, K. Hot and cold spot analysis using Stata. Stata J. 2016, 16, 613-631. [CrossRef]

64. Ludwig, C.; Hecht, R.; Lautenbach, S.; Schorcht, M.; Zipf, A. Mapping Public Urban Green Spaces Based on OpenStreetMap and Sentinel-2 Imagery Using Belief Functions. ISPRS Int. J. Geo-Inf. 2021, 10, 251. [CrossRef]

65. Guagliardo, M.F. Spatial accessibility of primary care: Concepts, methods and challenges. Int. J. Health Geogr. 2004, 3, 1-13. [CrossRef] [PubMed]

66. Jiao, H.; Li, C.; Yu, Y.; Peng, Z. Urban Public Green Space Equity against the Context of High-Speed Urbanization in Wuhan, Central China. Sustainability 2020, 12, 9394. [CrossRef]

67. Conners, R.W.; Trivedi, M.M.; Harlow, C.A. Segmentation of a high-resolution urban scene using texture operators. Comput. Vis. Graph. Image Process. 1984, 25, 273-310. 